\title{
An emerging picture of the seed desiccome: confirmed regulators and newcomers identified using transcriptome comparison
}

\author{
Emmanuel Terrasson ${ }^{1}$, Julia Buitink ${ }^{2}$, Karima Righetti ${ }^{2}$, Benoit Ly Vu ${ }^{3}$, Sandra Pelletier ${ }^{2}$, \\ Julia Zinsmeister ${ }^{3}$, David Lalanne ${ }^{2}$ and Olivier Leprince ${ }^{3 *}$ \\ ' Université d'Angers, UMR 1345 Institut de Recherche en Horticulture et Semences, SFR 4207 QUASAV, Angers, France \\ 2 Institut National de la Recherche Agronomique, UMR 1345 Institut de Recherche en Horticulture et Semences, SFR 4207 QUASAV, Angers, France \\ ${ }^{3}$ Agrocampus Ouest, UMR 1345 Institut de Recherche en Horticulture et Semences, SFR 4207 QUASAV, Angers, France
}

\section{Edited by:}

Jill M. Farrant, University of Cape

Town, South Africa

Reviewed by:

Ján A. Miernyk, University of

Missouri, USA

Vasileios Fotopoulos, Cyprus

University of Technology, Cyprus

*Correspondence:

Olivier Leprince, Agrocampus Ouest, UMR 1345 Institut de

Recherche en Horticulture et

Semences, 16 bd Lavoisier, 49045

Angers, France

e-mail: olivier.leprince@

agrocampus-ouest.fr
Desiccation tolerance (DT) is the capacity to withstand total loss of cellular water. It is acquired during seed filling and lost just after germination. However, in many species, a germinated seed can regain DT under adverse conditions such as osmotic stress. The genes, proteins and metabolites that are required to establish this DT is referred to as the desiccome. It includes both a range of protective mechanisms and underlying regulatory pathways that remain poorly understood. As a first step toward the identification of the seed desiccome of Medicago truncatula, using updated microarrays we characterized the overlapping transcriptomes associated with acquisition of DT in developing seeds and the re-establishment of DT in germinated seeds using a polyethylene glycol treatment $(-1.7 \mathrm{MPa})$. The resulting list contained 740 and 2829 transcripts whose levels, respectively, increased and decreased with DT. Fourty-eight transcription factors (TF) were identified including $M t A B / 3, M t A B / 5$ and many genes regulating flowering transition and cell identity. A promoter enrichment analysis revealed a strong over-representation of ABRE elements together with light-responsive cis-acting elements. In Mtabi5 Tnt1 insertion mutants, DT could no longer be re-established by an osmotic stress. Transcriptome analysis on Mtabi5 radicles during osmotic stress revealed that 13 and $15 \%$ of the up-regulated and down-regulated genes, respectively, are mis-regulated in the mutants and might be putative downstream targets of MtAB/5 implicated in the re-establishment of DT. Likewise, transcriptome comparisons of the desiccation sensitive Mtabi3 mutants and hairy roots ectopically expressing MtAB/3 revealed that 35 and $23 \%$ of the up-regulated and down-regulated genes are acting downstream of $M t A B / 3$. Our data suggest that $A B I 3$ and $A B I 5$ have complementary roles in DT. Whether DT evolved by co-opting existing pathways regulating flowering and cellular phase transition and cell identity is discussed.

Keywords: ABI3, ABI5, ABA, desiccation tolerance, transcription factor, seed, transcriptome, Medicago truncatula

\section{INTRODUCTION}

Understanding osmotic stress responses is one of the most important topics in plant science as this stress causes adverse effects on crop yield and quality (Hirayama and Shinozaki, 2010). For example, the recent drought waves that occurred in Europe in 2003 and in most of the USA in 2012 caused an estimated $30 \%$ reduction in primary productivity (Ciais et al., 2005; Claeys and Inzé, 2013). With the prospect of climate changes that are expected to worsen water limitations in the future, these figures suggest that major progress in understanding how plants cope with drought stress remains to be made. Given these agricultural challenges, it is remarkable that there exist biological mechanisms that allow complete desiccation with loss of viability (Hoekstra et al., 2001; Moore et al., 2008; Leprince and Buitink, 2010; Gechev et al., 2012; Gaff and Oliver, 2013). Desiccation tolerant seeds and leaves have acquired mechanisms that enable them to safely experience decreasing water potentials at which drought tolerant tissues are barely surviving (i.e., $>-3 \mathrm{MPa}$ for cell protoplasts). Therefore, it is argued that understanding the molecular processes leading to desiccation tolerance (DT) in seeds and resurrection plants might help the design and production of drought tolerant crops (Bartels and Sunkar, 2005; Moore et al., 2009; Rodriguez et al., 2010; Oliver et al., 2011; Gechev et al., 2012).

The desiccome can be referred to as the set of genes, proteins and metabolites that are required for DT (Leprince and Buitink, 2010). They are part of the protective mechanisms that can be grouped in at least three types that act synergistically (for reviews see Hoekstra et al., 2001; Tunnacliffe and Wise, 2007; Moore et al., 2008, 2009; Mönke et al., 2012; Leprince and Buitink, 2010; Gaff and Oliver, 2013): (1) protection by stabilization of membranes 
and proteins by non-reducing sugars, late embryogenesis abundant (LEA) proteins and heat shock protein (HSP), (2) protection against oxidative damage by a range of antioxidant compounds such as tocopherols, glutathione, together with a coordinated response of metabolism during drying and (3) protection against structural stresses imposed by drying such as cell wall modification, reorganization of endomembranes and cytoskeleton, vacuolization and chromatin condensation. The desiccome also includes the regulatory mechanisms and signaling pathways controlling the induction of these protective mechanisms leading to DT. However, these pathways are still poorly understood.

Abscisic acid $(A B A)$ has been long known to play a prominent regulatory role in DT. In several species of resurrection plants, exogenous ABA induces DT [reviewed by Gaff and Oliver (2013)] whereas in seeds, genetic screens for ABA insensitivity during germination has led to the discovery of abscisic acid insensitive 3 (abi3) mutants. Severe alleles of abi3 mutants of several species, including Arabidopsis (Ooms et al., 1993) and Medicago truncatula (Delahaie et al., 2013) produce desiccationsensitive seeds and have reduced expression of many genes related to DT like LEA genes. An ABI3 ortholog has been found to activate LEA genes in the moss Physcomitrella patens (Yotsui et al., 2013), suggesting that $\mathrm{ABI} 3$ is part of an evolutionarily conserved regulatory network. $A B I 3$ encodes a transcription factor belonging to the B3 domain-containing family and its function in the ABA signaling pathway in seeds is well characterized (Cutler et al., 2010; Hauser et al., 2011; Nakashima and Yamaguchi-Shinozaki, 2013). Transcriptional targets of ABI3 have been identified based either on transcript analysis in lossof-function mutants or over-expressing transgenics (Nakashima et al., 2006) or through genome wide chromatin immunoprecipitation (Mönke et al., 2012). They include oleosins, LEA proteins and storage proteins. However, the experimental set up of these studies did not consider a putative link with DT.

To date, the ABA-signaling pathway leading to DT is mainly inferred from the knowledge gained from genetic and biochemical studies in drought tolerance in vegetative tissues and seed development. Indeed, the backbone of the ABA core signaling pathway, including positive and negative regulators involved in the response to osmotic stress in vegetative tissues, is very similar to that involved in seed maturation during which DT is acquired (Finkelstein et al., 2005; Fujita et al., 2011; Hauser et al., 2011; Nakashima and Yamaguchi-Shinozaki, 2013). In seeds and seedlings, basic leucine zipper (bZIP) transcription factors (TF) belonging to the ABA Responsive Element Binding Factors (AREB/ABF) and ABA INSENSITIVE 5 (ABI5) clade represent key TF controlling ABA-responsive gene expression such as LEA genes by interacting with $\mathrm{ABA}$ responsive regulatory elements (ABRE) in their promoter region. In Arabidopsis, promoters of genes with transcript levels that accumulate during maturation drying and that are stored in the dry seeds exhibit an enrichment in ABRE elements (Nakabayashi et al., 2005). ABI5 is considered as a crucial player in ABA signaling (Fujita et al., 2011). ABI5 appears to be courted by multiple proteins, thereby forming a complex interactome (Lindemose et al., 2013). There is also a large body of evidence suggesting that ABI5 forms a complex with $\mathrm{ABI} 3$ to regulate the expression of downstream genes with
ABI3 acting as an accessory enhancer of transcription (Nakamura et al., 2001; Lindemose et al., 2013). It is suggested that such interaction occurs only during seed maturation (Finkelstein et al., 2005). ABI5 exerts a positive regulation of EM1 and EM6 genes (Nakamura et al., 2001; Finkelstein et al., 2005) both encoding LEA proteins whose abundance correlates with DT in developing and germinating seeds of Medicago (Boudet et al., 2006; Chatelain et al., 2012). However, null alleles of ABI5 apparently produce desiccation-tolerant seeds in Arabidopsis. During seedling establishment, ABI5 controls an ABA- or osmotic stress induced post-germinative growth arrest that is accompanied by an increased drought tolerance (Lopez-Molina et al., 2001, 2002). Therefore, the precise role of ABI5 in DT, if any, remains to be elucidated.

In this study, we investigated the regulatory components involved in DT in M. truncatula seeds by revisiting the transcriptome changes associated with the acquisition of DT during seed maturation and the re-establishment of DT in emerged radicles upon an osmotic treatment using a PEG solution at $-1.7 \mathrm{MPa}$ (Buitink et al., 2006; Verdier et al., 2013). We used an updated Nimblegen slide containing the almost complete $M$. truncatula genome. One of the identified TF that was further characterized for its role in DT was MtABI5. New putative ABI5-regulated target genes that are involved in DT were identified from a transcriptome analysis. In addition, to identify ABI3-related genes involved in DT, we also took advantage of recently obtained transcriptome data on Medicago Mtabi3 seeds and hairy roots ectopically expressing MtABI3. The comparison of these five transcriptomes provides insights into the desiccome that is regulated by $\mathrm{ABI} 3$ and $\mathrm{ABI} 5$ and identifies new regulators that might play a role in DT in seeds.

\section{MATERIALS AND METHODS PLANT MATERIALS AND PHYSIOLOGY}

Plants of Medicago truncatula ssp. tricycla (R108) were grown in a sterile mix of vermiculite and soil in a growth chamber at $24^{\circ} \mathrm{C} / 21^{\circ} \mathrm{C}, 16 \mathrm{~h}$ photoperiod at $200 \mu \mathrm{M} \mathrm{m} \mathrm{m}^{-2} \mathrm{~s}^{-2}$. Seeds were harvested upon pod abscission and stored at $5^{\circ} \mathrm{C}$ at $43 \%$ relative humidity $(\mathrm{RH})$ until use. Two $M$. truncatula mutants with Tnt1 insertions in the MtABI5 gene (NF4383, hereafter referred to as Mtabi5-1 and NF3376, Mtabi5-2) were obtained from the Samuel Noble Foundation (Oklahoma, USA). Tnt1 insertions in both mutants were verified by PCR according to Delahaie et al. (2013) using forward and reverse primers ATGGTGGTAAGAGAAGGTGAGAT and AGCAGCAAGATCTAGAGCCAGA, respectively. Mutant and wild-type lines (R108) were multiplied in a growth chamber according to Chatelain et al. (2012). The Mtabi5-1 line was backcrossed twice.

To determine ABA sensitivity, batches of 30-50 seeds were scarified with sand paper and imbibed on filter paper on a range of ABA concentrations (mixed isomers, Sigma, St Louis, MO, USA) at $20^{\circ} \mathrm{C}$ in the dark. ABA was dissolved in methanol prior to dilution in water. Control seeds were imbibed in the $\mathrm{MeOH}$ concentration corresponding to the highest ABA concentration $(0.5 \% \mathrm{MeOH})$. Germination was scored after 14 days. 
The re-establishment of DT after germination was performed according to Buitink et al. (2003). Germinated seeds with a protruded radicle length of $2.7-2.9 \mathrm{~mm}$ (thereafter referred to as $2.7 \mathrm{~mm}$ ) were selected and submitted to an osmotic treatment by incubation in a PEG 8000 solution $(-1.7 \mathrm{MPa})$ at $10^{\circ} \mathrm{C}$ in the dark. After $72 \mathrm{~h}$, seeds were removed from the PEG solution and rinsed thoroughly. Three replicates of 50 radicles before and after PEG treatment from WT and Mtabi5 mutants were excised and frozen for RNA extraction. DT of germinated, untreated and PEG-treated germinated seeds was assessed by drying 50 seeds for each condition for 3 days at $20^{\circ} \mathrm{C}$ under an air flow at $42 \% \mathrm{RH}$. Radicles were considered desiccation-tolerant when they resumed growth upon re-imbibition.

\section{BIOINFORMATIC ANALYSES}

A blast was performed on the Medicago IMGAG Mt3.5.1 version using the Arabidopsis ABI5 amino acid sequence (At2g36270.1) to retrieve homologous genes (score $e<1 \mathrm{E}-20$ ). An unrooted tree was constructed with the nine identified MtABI5-like sequences and the Arabidopsis ABI5 using the sequence alignment program ClustalW (http://www.ebi.ac.uk/Tools/msa/clustalo/) and presented using Treeview (Page, 1996). Gene lists were analyzed for enrichment in gene ontology (GO) terms using the Singular Enrichment Analysis (SEA) tool of AgriGO (http://bioinfo.cau. edu.cn/agriGO/) with a Chi-square statistical test method and the Yekutieli multi-test adjustment method (Du et al., 2010). For the large dataset of down-regulated transcripts related to DT, Plant Slim GO analysis was used. Data lists were analyzed using the Arabidopsis TAIR9 background based on homology with the Medicago sequences since the $M$. truncatula genome background in AgriGO is incomplete.

For cis-element enrichment analysis of the DT-UP list, $1.5 \mathrm{~Kb}$ promoter regions upstream of the translational start of the 382 IMGAG Mt3.5.1 sequences were retrieved using the Legoo gateway (https://www.legoo.org/). Promoter sequences were analyzed using the PLACE database resource, (http://www.dna.affrc.go.jp/ PLACE/signalup.html). The frequency of a specific motif in the promoters of genes was tested against a background frequency generated using a randomized sample of 1470 Medicago genes. The significance of the enriched cis-elements was calculated using a Chi-square test against the background sample.

\section{RNA EXTRACTION AND MICROARRAY ANALYSIS}

Total RNA were extracted using the nucleospin RNAplant kit (Macherey Nagel, Düren, Germany) and 400 ng were amplified using the Ambion messageAmp II (Ambion, Austin TX) following manufacturer's instructions. Five $\mu \mathrm{g}$ of amplified RNA were retro-transcribed with $400 \mathrm{U}$ of Superscript II reverse-transcriptase (Invitrogen Corp., Carlsbad, CA) and labeled with $1.5 \mathrm{mmol}$ of Cyanine-3 (Cy3) or Cyanine-5 (Cy5) (Interchim, France) then purified using NucleoSpin Gel and PCR Clean-up column kits (Macherey-Nagel, GmbH and Co. KG, Germany). Purified and labeled cDNA were quantified using a NanoDrop ND-1000. Corresponding Cy3- and Cy5 labeled samples $(30 \mathrm{pmol})$ were combined and co-hybridized to Medtr_v1.0 $12 x 135 \mathrm{~K}$ arrays according to Verdier et al. (2013). The Medtr_v1.0 chip was in situ synthesized by Nimblegen (Madison, WI) and contains 102,123 60-mer oligoprobes that were designed from an intermediate annotation of the $M$. truncatula genome, containing one probe per sequence of the IMGAG Mt3.5.1 version and additional sequences from RNAseq (Verdier et al., 2013). Three biological replicates were analyzed per comparison using the dyeswitch method, and statistical analysis on the gene expression data was performed according to Verdier et al. (2013). Probes with a $P<0.01$ and a $\log$ ratio $>|1|$ were considered differentially expressed.

\section{DATA SUBMISSION}

Nimblegen microarray data were deposited on the NCBI GEO database [(Barrett et al., 2012); accession GSE51830]. Data on the ectopic expression of $M t A B I 3$ in hairy roots discussed in this publication have been deposited in NCBI Gene Expression Omnibus (Edgar et al., 2002) and are accessible through GEO Series accession number GSE44291 (http://www.ncbi.nlm.nih. gov/geo/query/acc.cgi?acc=GSE44291).

\section{QUANTITATIVE PCR}

For real time RT-PCR, $1 \mu \mathrm{g}$ total RNA was reverse-transcribed using Quantitect Reverse Transcription kit (Qiagen, Courtaboeuf, France) and qPCR was performed on a CFX96 Real-Time Detection System (Bio-Rad Laboratories, Hercules, CA) using the manufacturer's instructions with SsoFast Eva Green Supermix (Bio-Rad Laboratories, Hercules, CA). For Medtr5g010210.1 (GCL210, glutamate cysteine ligase), 5'TCTACGGCCAAAACTG TTGC3' and 5'CGTAACGTACCTTGGGCACTA3' were used as forward and reverse primers, respectively. For Medtr5g010230.1 (GCL230), 5'TCCTGGATATGATTGCTGATTGGA3' and 5'AG AGTCTTTAAACCGGTGAAAGGA3' were used as forward and reverse primers, respectively. The reference gene used for normalization was MSC27 (Bolingue et al., 2010). Relative expression levels were calculated using the comparative $2 \triangle(\mathrm{Ct})$ method. Each data point represents the mean of three biological replicates.

\section{RESULTS AND DISCUSSION IDENTIFICATION OF THE TRANSCRIPTIONAL DESICCOME USING TWO PHYSIOLOGICAL MODELS OF DESICCATION TOLERANCE}

To obtain an overview of the genes implicated in DT, we produced two transcriptome data sets using an updated Medicago Nimblegen microarray: one on the acquisition of DT during maturation (GEO database accession GSE49350), and a second one on the re-establishment of DT during PEG incubation. To identify genes that are differentially expressed during the acquisition of DT, transcript levels were compared between 14 days after pollination (DAP), when seeds are still desiccation sensitive, and 32 DAP, when $100 \%$ of the seeds have acquired their DT (Figure 1A). Data analysis identified 3626 and 14601 transcripts that increased or decreased at least two-fold, respectively, between 14 and 32 DAP. Next, we characterized the transcriptome in relation to the re-establishment of DT. DT is lost following germination after the radicle is protruded, first in the radicles and then in the cotyledons. However, it is possible to re-establish DT in these protruded radicles by an osmotic stress treatment using a PEG solution at $-1.7 \mathrm{MPa}$ (Buitink et al., 2003; Faria et al., 2005). We performed a transcriptome analysis on $2.7 \mathrm{~mm}$ 

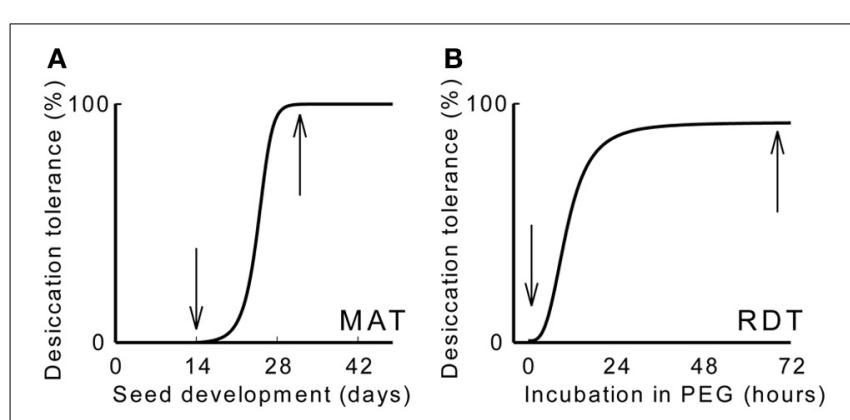

C

UP

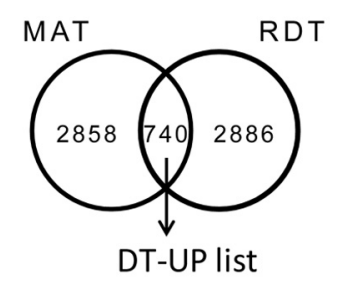

FIGURE 1 | Changes in desiccation tolerance (DT) and the associated desiccome in Medicago truncatula. (A,B) Schematic representation of the acquisition of DT during seed maturation (MAT) at the indicated days after pollination (DAP) [A, curve taken from Chatelain et al. (2012)] and the re-establishment of DT (RDT) in emerged radicles of germinated seeds during the incubation in a polyethylene glycol solution (PEG) at $-1.7 \mathrm{MPa}$ [B, curve taken from Buitink et al. (2006)]. Arrows indicate the time at which samples were taken for the transcriptome analysis. (C) Venn diagrams comparing the overlapping transcripts whose levels are increased (UP) or decreased (DOWN) during the transition from a desiccation-sensitive to -tolerant stage during maturation (MAT) and PEG incubation (RDT). Genes were considered statistically different when $-1<$ $\mathrm{M}(\log 2)>1$ and $P<0.01$.

long protruded radicles before and after $72 \mathrm{~h}$ of PEG incubation, corresponding to desiccation sensitive and -tolerant tissues, respectively (Figure 1B). A total of 3598 and 6691 transcripts increased or decreased significantly (ratio $\log 2>1$ or $<-1, P<$ 0.01 ). Both datasets were compared to each other to identify overlapping transcripts (Figure 1C). This resulted in the identification of 740 transcripts that positively correlate with DT (hereafter referred to as the DT-UP list) and 2829 transcripts that are downregulated when DT is installed (DT-DOWN list) (Figure 1C) (Supplementary Table S1). It should be noted that some redundancy exists between the probes. Some of them are recognized by the same gene transcript, which can only be removed when the assembly of the M. truncatula genome is completed.

The DT associated transcriptome of Medicago corresponding to a more complete dataset using updated slides, was compared to the recently obtained data set on a similar system of re-establishment of DT in Arabidopsis (Maia et al., 2011). Compared to this study, the number of transcripts overlapping between Arabidopsis and Medicago increased from 22 (48 transcripts) to $77 \%$ (115) of the total amount of up-regulated genes in Arabidopsis during re-establishment of DT and from 13 (49 transcripts) to $50 \%$ (139) for the down-regulated genes. These values reinforce the idea that core mechanisms and key regulators involved in DT are conserved across species, despite the fact that whole seeds were used for Arabidopsis and only radicles for M. truncatula (Maia et al., 2011).

To gain insight into the Medicago DT-related transcriptome, a GO enrichment analysis was performed using agriGO. Within the up-regulated genes, the main enriched GO terms belonged to three broad categories: developmental processes, endogenous stimuli and external stimuli and stress (Figure 2A). A closer look at these GO terms identified categories previously described by Maia et al. (2011) in the relation to the re-establishment of DT in germinated seeds, such as post-embryonic development, response to $\mathrm{ABA}$, and response to water deprivation. The latter two GO terms were also enriched in the transcriptome of dehydrating leaves of the resurrection plant Craterostigma plantagineum (Rodriguez et al., 2010), reinforcing the importance of ABA as a core regulator of DT. Our data also revealed an important and previously un-described category in the DT-UP list, namely "anatomical structure development." Apart from genes that have been discovered previously in relation to DT, such as LEA genes, oleosins, or 1-cys peroxyredoxin, this GO category revealed genes whose Arabidopsis homologs are involved in the regulation of cell growth, cell formation or identity. This category also contains several probes whose homologs in Arabidopsis impose maternal effects on seed development such as DEMETER (responsible for endosperm maternal-allele-specific hypomethylation at the MEDEA gene) and MATERNAL EFFECT EMBRYO ARREST 11 and 14 (MEE11, MEE14), whose mutants are embryo lethal because of endosperm development arrest (Pagnussat et al., 2005). It is long recognized that many seed traits such as weight, germination and even longevity (i.e., survival in the dry state for a period of time) are under the influence of parent-of-origin effects (Blödner et al., 2007; Donohue, 2009; Kochanek et al., 2011). Since the acquisition of DT is essential to complete the plant life cycle, these data raise the intriguing question whether maternal effects might also influence the transcriptional part of the desiccome.

The GO categories of the DT-down list were related to cell cycle, growth and regulation of cell size (Figure 2B). For instance, they contained genes associated with the modification of cell walls (cellulose synthase, expansins, ATGH9B1 (ARABIDOPSIS THALIANA GLYCOSYL HYDROLASE 9B1) and the cytoskeleton. Also GO categories related to transport and metabolism were found to be enriched in the down-regulated desiccome.

\section{CHARACTERIZATION OF TRANSCRIPTIONAL REGULATORS AND REGULATORY Cis-ELEMENTS IN THE UP-REGULATED DESICCATION-TOLERANCE RELATED TRANSCRIPTOME}

To gain further insight into the transcriptional machinery regulating DT independently of the developmental context, $48 \mathrm{TF}$ that were manually curated using Ensembl and Plantdtb (http:// planttfdb.cbi.pku.edu.cn) were retrieved from the up-regulated gene list (Table 1). AP2/EREBP, bHLH, and bZIP family members were found to be the most abundant TF. Twenty-two percent of these TF correspond to Arabidopsis homologs associated with freezing, cold and drought response and/or mediating an ABA response such as members of the DREB (DRE binding protein) subfamilies (Table 1, bold characters) as previously described (Fujita et al., 2011). 


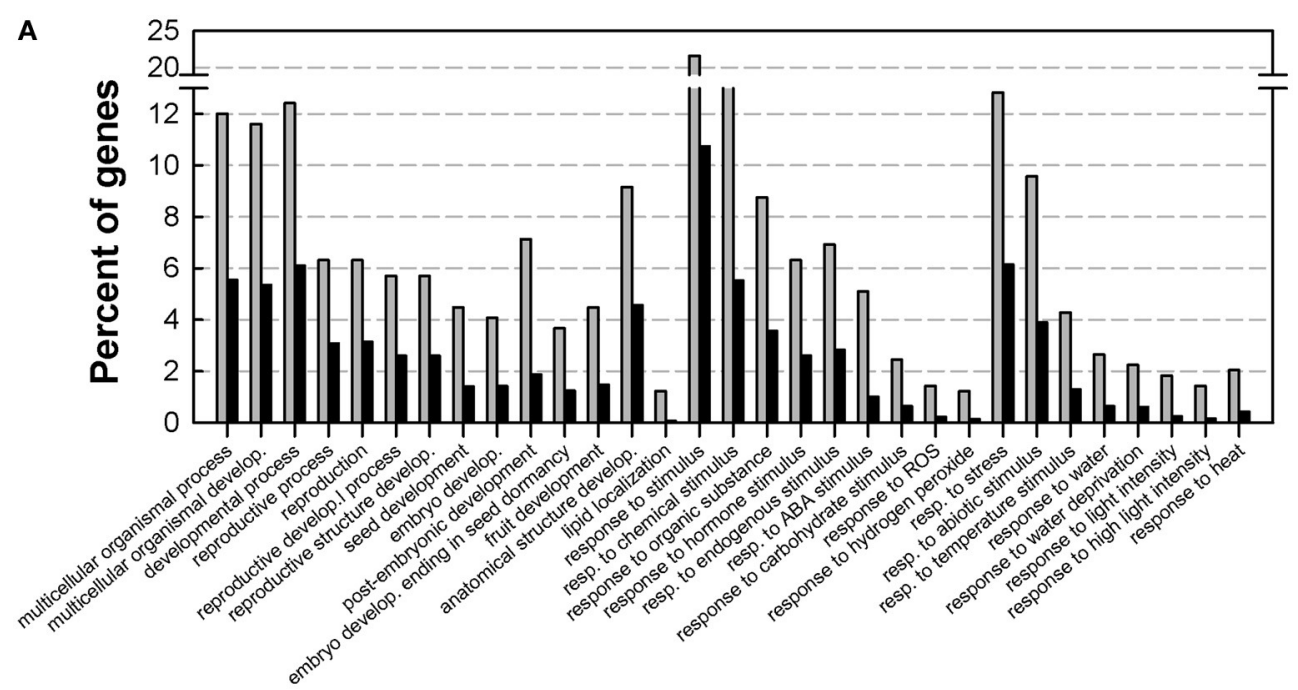

B

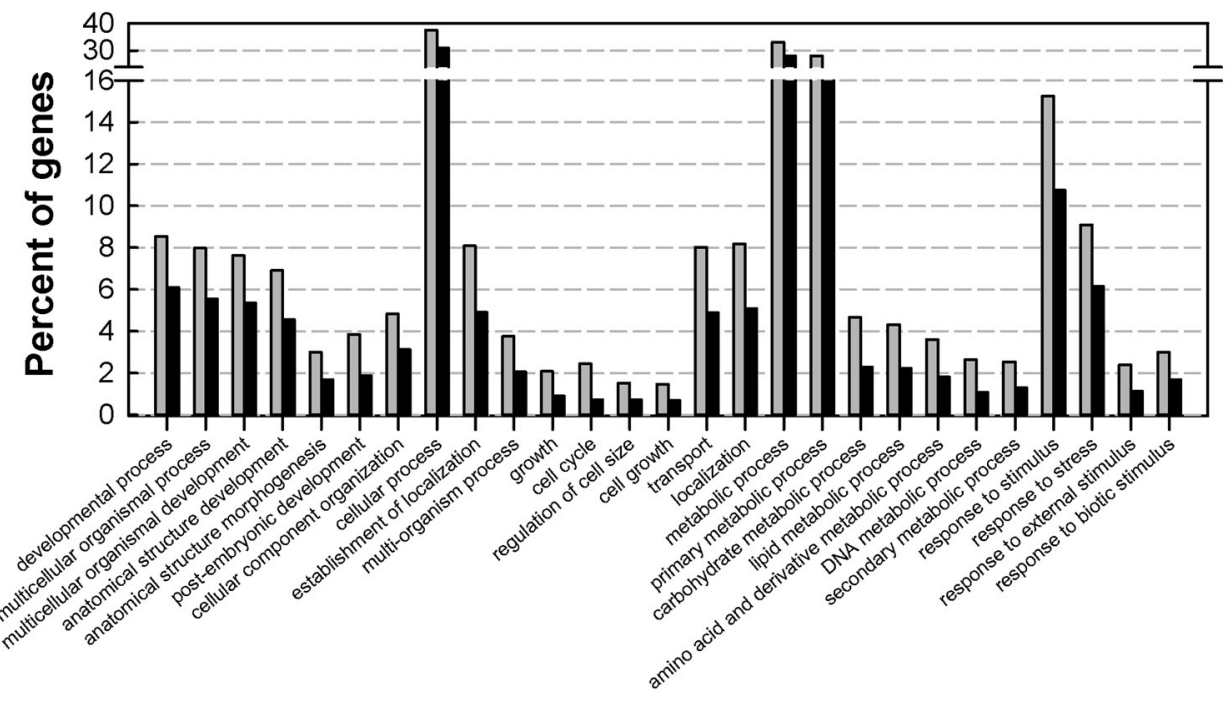

GO terms

FIGURE 2 | Gene Ontology (GO) enrichment analysis of the desiccation tolerant UP list (A) and desiccation tolerant DOWN list (B). Analysis was performed on the DT-UP and DT-DOWN list from Figure 1 using AgriGO with the Arabidopsis TAIR9 background applying a $\mathrm{Chi}^{2}$ statistical test with the Yekutieli multi-test adjustment method. Gray bars: input genes, black bars background genes.
Strikingly, 17 TF (35\%) with increased transcript levels in relation to DT were homologs of Arabidopsis regulators that are broadly associated with cell fate or cell identity (see italics characters in Table 1). For example, TRANSCRIPTION FACTOR GROUP E6 (GTE6) regulates differences in leaf patterning between juvenile and mature leaves. PLETHORA 2 (PLT2) is a master regulator of embryonic pattern formation during embryogenesis and root development during seedling growth (Galinha et al., 2007). CUP-SHAPED COTYLEDON 3 (CUC3) regulates embryonic shoot meristem formation and cotyledon boundary specification (Hibara et al., 2006). HAT3 controls apical embryo development and meristem function (Turchi et al., 2013). GRF5 regulates the promotion and/or maintenance of cell proliferation activity in leaf primordia. In rice, the homolog of WOX11 regulates the activation of crown root emergence and growth
(Zhao et al., 2009). Yet, during the acquisition of DT during maturation and during PEG, the cell cycle activities are repressed (Faria et al., 2005). Whether these TF participate at the repression of these activities or whether they are necessary to coordinate the resumption of growth and development during rehydration remains to be assessed.

The Medicago TF list also contains homologs of transcriptional activators of Arabidopsis that participate in the precise regulation of the timing of flowering by incorporating temperature or day length information to promote floral transition and flower meristem identity (Table 1). Among these, we find bHLH80 and bHLH130, respectively, homologs of FLOWERING BHLH 1 and 4 (FBH1 and FBH4), MADS-box protein SVP (homolog of SHORT VEGETATIVE PHASE SVP) and Myb-related protein MYB4 (homolog of MYB17) (Gregis et al., 2008; Jang et al., 

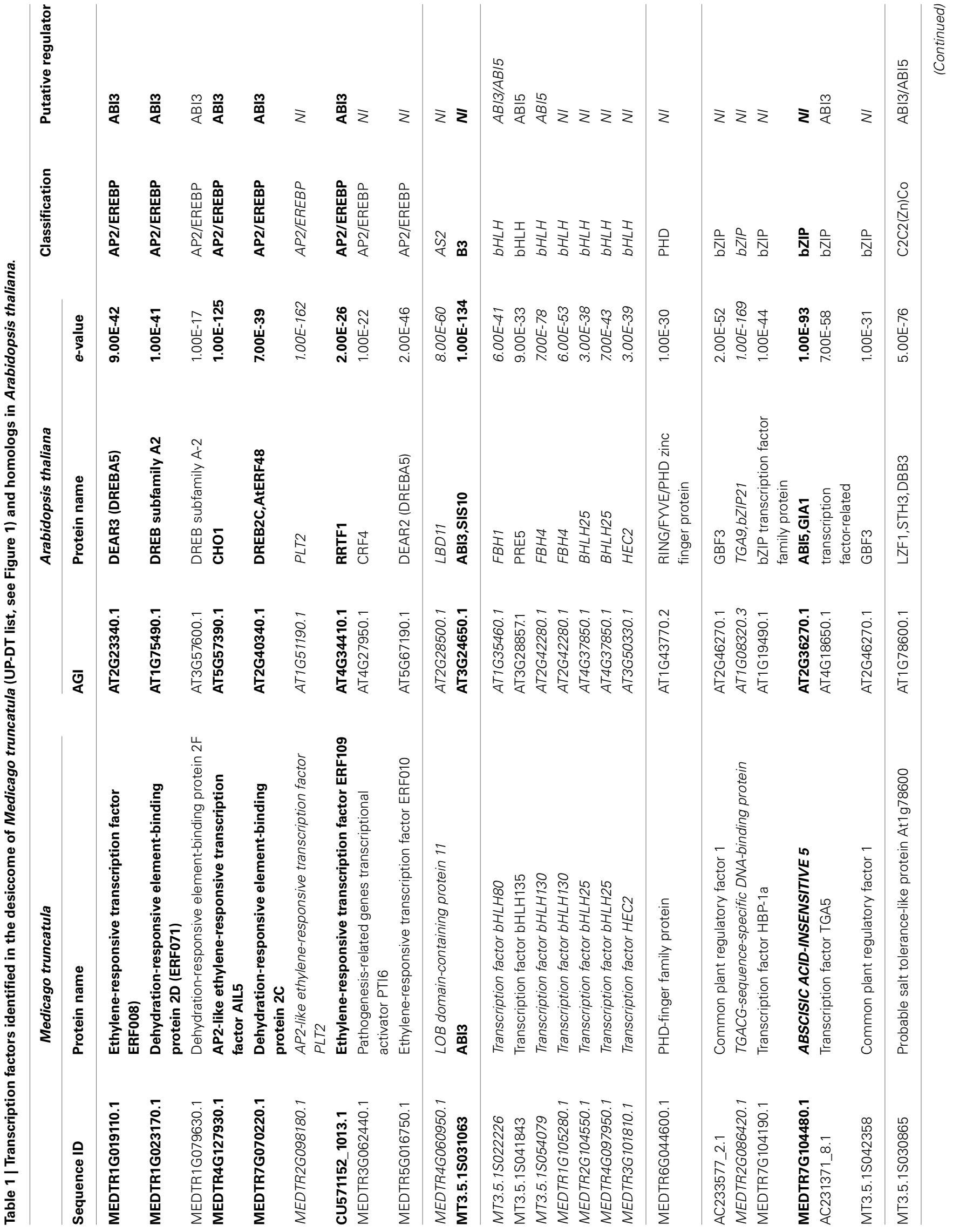


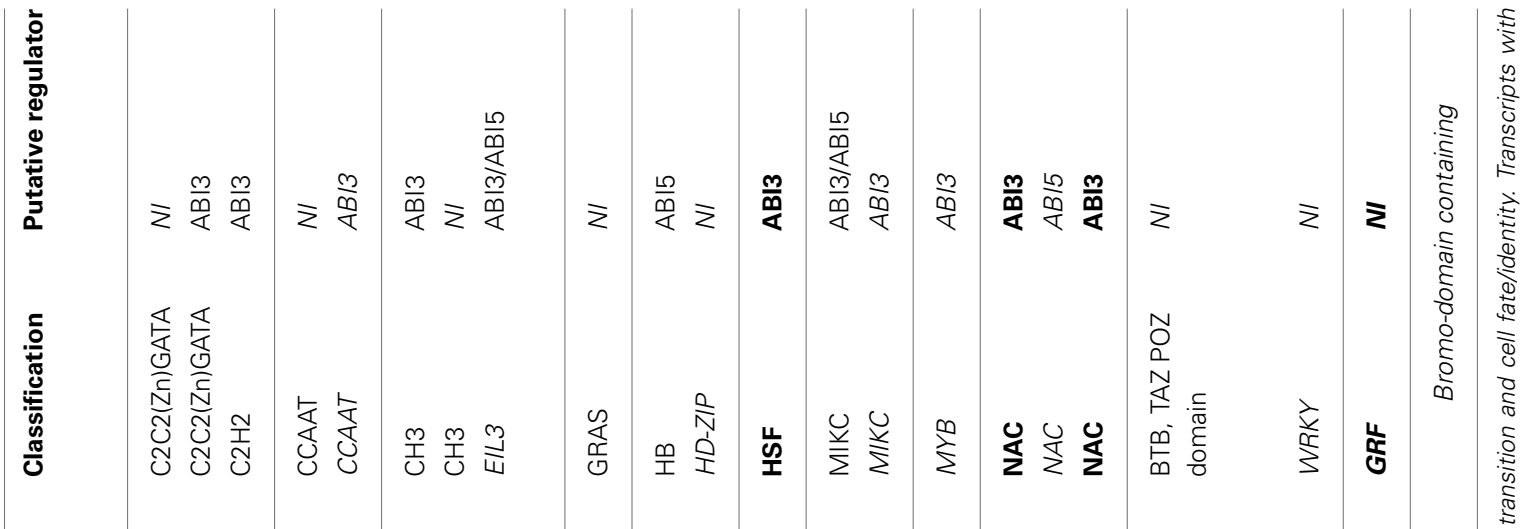

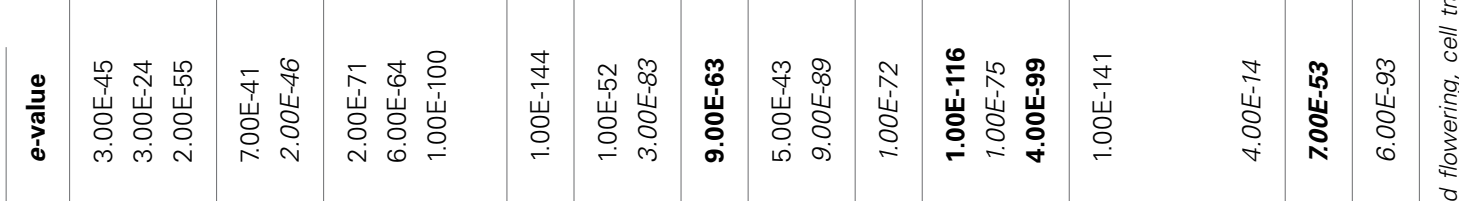
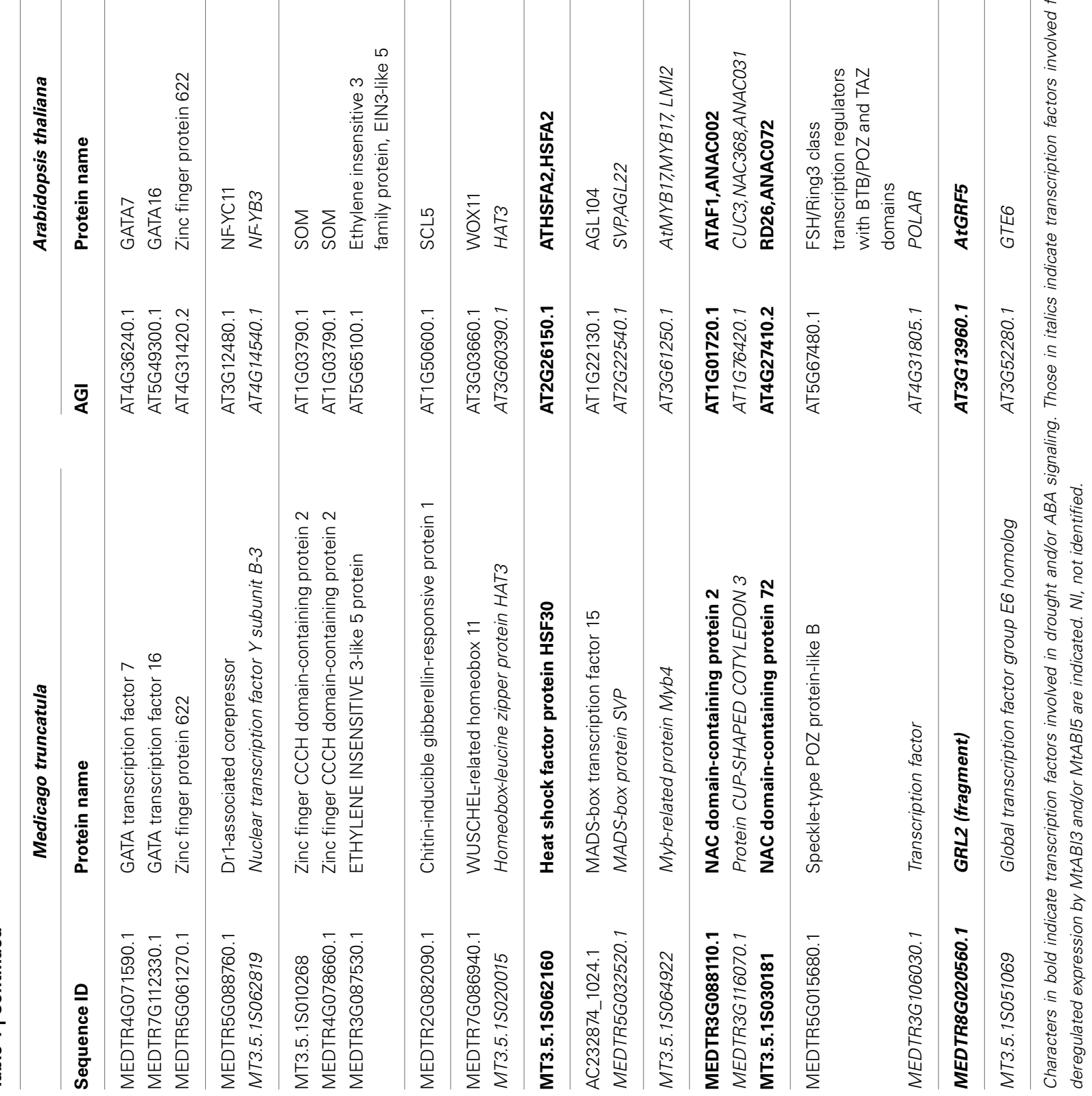
2009; Zhang et al., 2009; Ito et al., 2012). The implication of regulators integrating light information or controlling flowering was further reinforced by the identification of homologs of CHO1 (CHOTTO1) and SOM (SOMNUS) (Kim et al., 2008; Yano et al., 2009) and, in the DT-UP list, the photoreceptor FLAVIN-BINDING KELCH REPEAT F BOX 1(FKF1). Although our analysis is based solely on the comparison of transcript levels, and care should be taken with the interpretation of the data based on Arabidopsis homologs, altogether, these findings raise new questions about the mechanisms involved in the regulation of DT and developmental transitions. One such question is whether these regulators could play a role in integrating developmental and environmental signals such as light to maintain irreversible the transition from a desiccation-sensitive to a tolerant stage.

If DT has integrated regulatory pathways that relay developmental and environmental cues, then this should be revealed in the cis-regulatory elements of promoter genes of the DT-UP list. For 393 out of 740 transcripts of the DT-UP list, promoters could be retrieved using the IMGAG Mt3.5.1 version. The most abundantly represented cis-elements were related to ARBRE elements (Table 2): 213 out of 393 promoters contained ABRE-related sequences (ABRERATCAL). These elements are targets of the bZIP ABI5 or ABRE binding factors, which suggests a role of these TF in DT in M. truncatula seeds. Many promoters of the DT-UP genes also contain DRE elements, but they were not significantly over-represented. DRE regulatory ciselements known to regulate ABA-dependent and -independent gene expression in response to osmotic stress and their role in seeds has been suggested (Fujita et al., 2011). Numerous genes contained cis-elements that are related to light mediated regulation/“G box" (GBOXLERBCS), known for binding with GBF, "BoxII” (LREBOXIIPCCHS1), "LRE” (LRENPCABE), and "Sequences Over-Represented in Light-Induced Promoters" (SORLIP4AT). The cis-element GBOXLERBCS was also found enriched in the DT transcriptome of PEG-treated germinated seeds of Arabidopis (Maia et al., 2011), and supports our hypothesis that regulation of DT has adopted regulatory pathways integrating light signals. Two overrepresented cis-regulatory elements were related to auxin, AUXREPSIAA4 and SGBFGMGMAUX28. This is consistent with the observation that several genes involved in cell identity present in the DT-list (PLT2, GRF5, Table 1) mediate auxin responses. Twenty seven genes contained the Sucrose Responsive Element 2 (SURE2), a motif conserved among genes regulated by sucrose (Grierson et al., 1994). Finally, 27 genes contained a UPRMOTIFIIAT "Motif II," a conserved UPR (unfolded protein response) cis-acting element in Arabidopsis genes coding for stress proteins (Martinez and Chrispeels, 2003, Table 2).

\section{A NEW PLAYER IN DESICCATION TOLERANCE: THE bZIP TRANSCRIPTION FACTOR MIABI5}

One of the TF that was present in the DT-UP list is a bZIP that is highly homologs to the Arabidopsis ABI5 gene (Table 1). In Arabidopsis, ABI5 binds to ABRE elements of Em genes whose homologs were found in our DT list (Finkelstein et al., 2005; Cutler et al., 2010; Supplemental Table S1). In concert with ABI3, ABI5 is known to regulate post-germinative growth arrest leading

Table 2 | Indentification of Cis-acting elements in promoters of genes correlated with desiccation tolerance in Medicago truncatula.

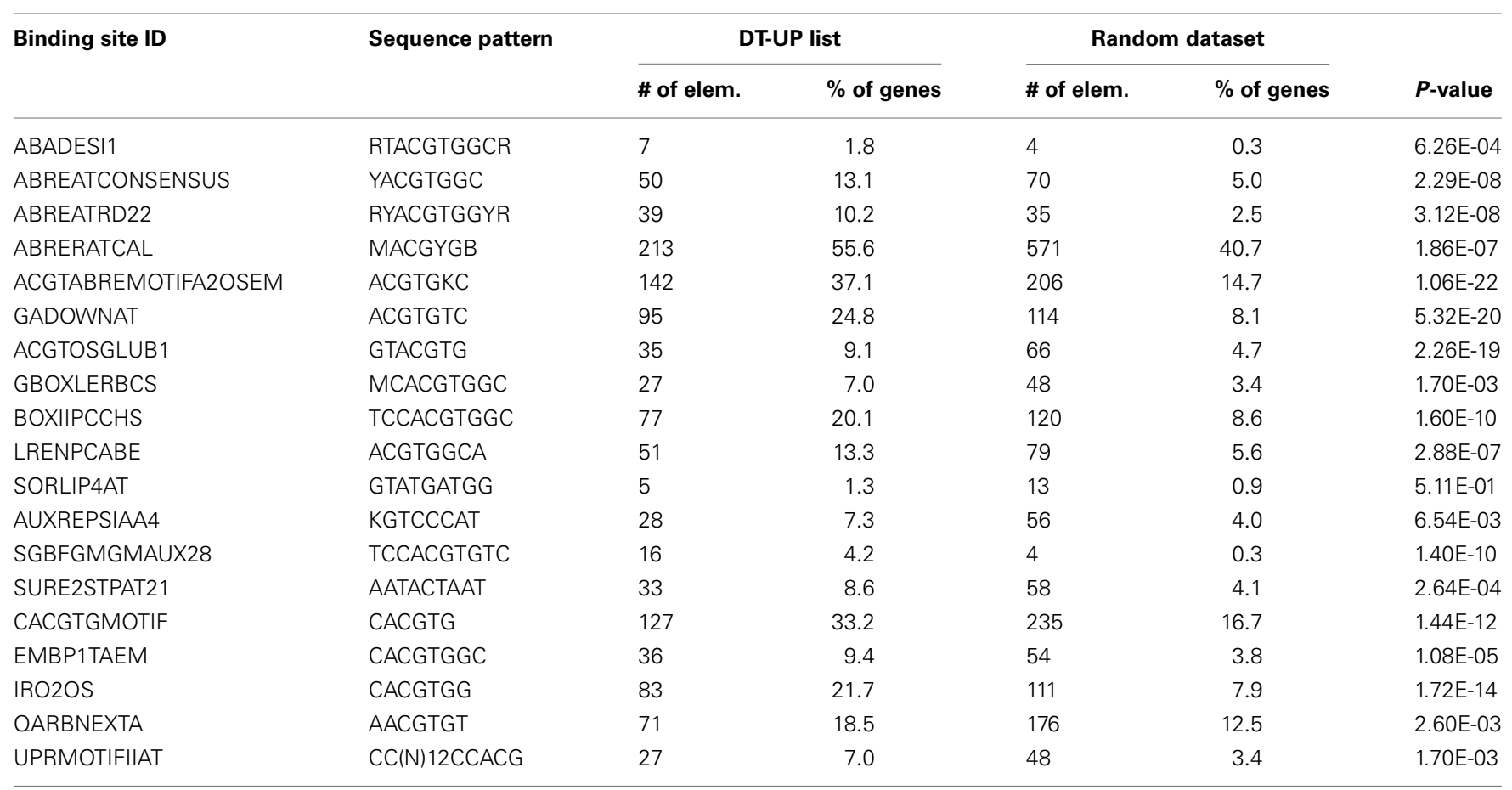

Promoter enrichment analysis of 382 up-regulated genes from the desiccome (DT-UP list, Figure 1) using 1.5Kb promoter regions upstream of the translational start. DNA motifs were counted using PLACE database resource (http://www.dna.affrc.go.jp/PLACE/signalup.htm/).\#, mumber of cis-elements in the indicated data sets. 
to drought tolerance in Arabidopsis seedlings (Lopez-Molina et al., 2001, 2002). This prompted us to test whether MtABI5 was a key factor in regulating DT and if so, what its target genes are in the desiccome gene set. Considering that the Arabidopsis genome comprises nine genes forming a $A B I 5$-homologs subfamily of bZIP TF binding to ABRE elements (Fujita et al., 2011), we first determined whether the Medicago gene Medtr7g104480 is homologous to ABI5 or other ABI5-like genes. The Arabidopsis ABI5 amino acid sequence was blasted against the Mt3.5.1 Medicago database and nine sequences were retrieved. A phylogenetic tree was constructed including the Arabidopsis ABI5 sequence (Figure 3A) and confirmed that Medtr7g104480 is indeed the closest homolog to ABI5.

The function of MtABI5 in DT was assessed using two independent homozygous Tnt1 insertion mutants (Mtabi5-1 and Mtabi5-2, Figure 3B) that were obtained from the insertion collection curated by the Samuel Noble Foundation. The Tnt1 insertions were located at 599 and 1034 bp from the start-codon, respectively, before the bZIP domain (Figure 3B). After the production of freshly harvested seeds, the ABA sensitivity of both Mtabi5 mutants was tested (Figure 3C). Like for abi5 mutants of Arabidopsis (Lopez-Molina et al., 2001), mature Mtabi5 seeds exhibited a strongly reduced sensitivity to ABA. In the presence of $10 \mu \mathrm{M}$, Mtabi5 seeds germinated at $100 \%$ whereas germination was fully inhibited in wild type seeds at this concentration.

Scarified, dry mature seeds of the Mtabi5 mutants were all able to germinate when imbibed at $20^{\circ} \mathrm{C}$ on wet filter paper, indicating that they acquired DT. Next, we tested whether ABI5 was implicated in the re-establishment of DT in sensitive emerged radicles during an osmotic treatment at $-1.7 \mathrm{MPa}$ using a PEG solution. In germinated wild type seeds, DT of emerged radicles is lost progressively during growth from 1 to $3 \mathrm{~mm}$ (Buitink et al., 2003). Control experiments confirmed that germinated seeds of the Mtabi5 mutants were not desiccation tolerant (Figure 4A). Furthermore, like germinated untreated wild-type seeds, the germinated Mtabi5 mutants started to lose their viability when water content decreased below $1.0 \mathrm{~g} \mathrm{H}_{2} \mathrm{O} \mathrm{g} \mathrm{DW}-1$ and decreased sharply below $0.5 \mathrm{~g} \mathrm{H}_{2} \mathrm{Og} \mathrm{DW} \mathrm{gW}^{-1}$ (Figure 4B). The comparable critical water content between germinated Mtabi5 and wild type seeds suggests that Mtabi5 seeds are not more sensitive to drought. Next, we tested whether germinated Mtabi5 seeds exhibiting a $2.7 \mathrm{~mm}$ long emerged radicle were able to tolerate the osmotic stress $(-1.7 \mathrm{MPa})$ brought by the PEG treatment that is necessary to re-establish DT in wild type seeds. Figure 4A shows that after 3 days in PEG, germinated Mtabi5 seeds were able to resume growth when transferred into water. However, when germinated, PEG-treated Mtabi5 seeds were dried to water contents of 9\% (dry weight basis), they were no longer able to resume growth upon rehydration, indicating that the Mtabi5 seeds remain desiccation sensitive, and lost their competence to re-establish DT upon osmotic treatment (Figure 4A).

Our data show that Mtabi5 seeds acquire their DT during maturation but were unable to re-establish it after germination. Although the temporal acquisition of DT might differ between Mtabi5 seeds and wild type seeds during seed development, our data suggest that MtABI5 is much more a critical determinant for

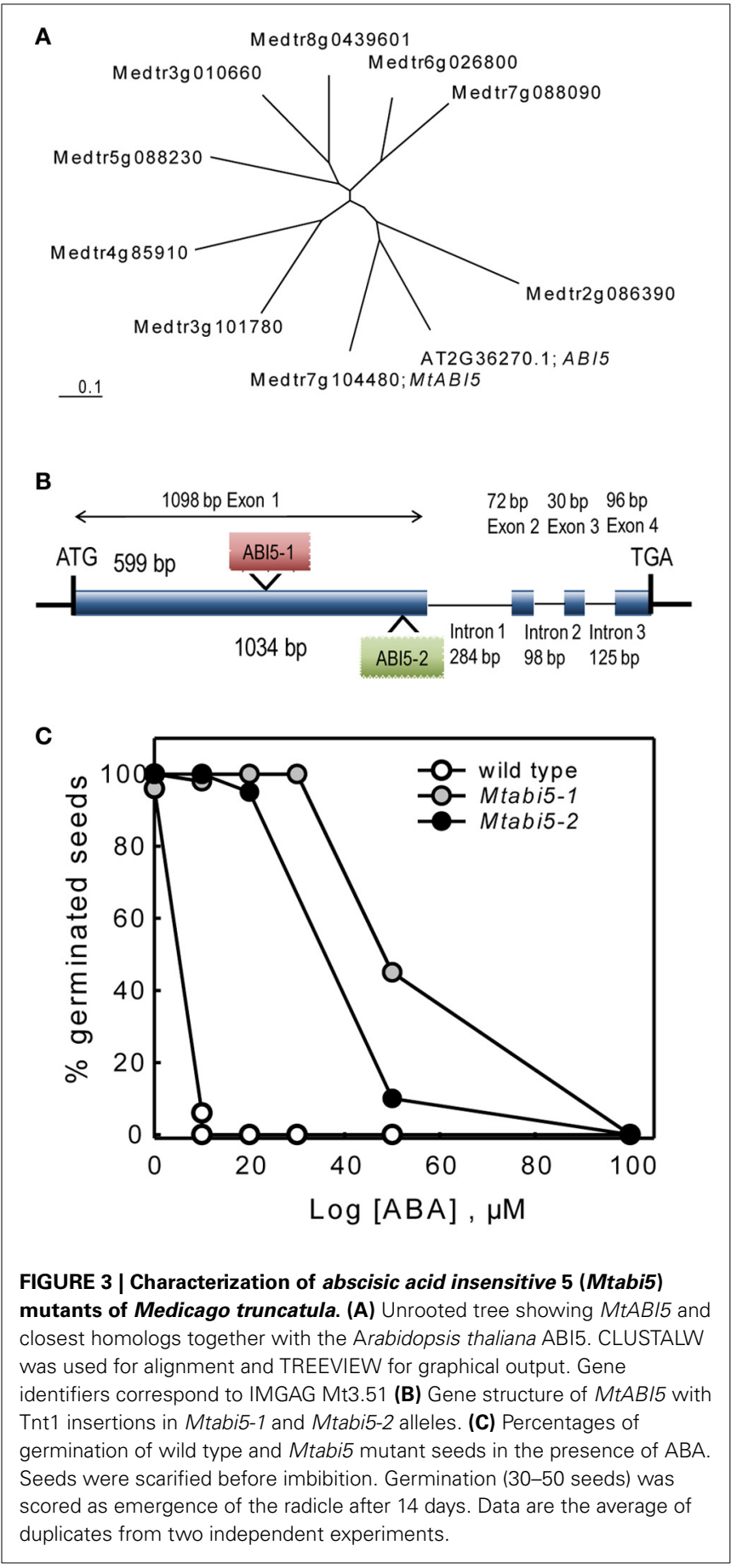

DT in seedlings than during maturation. Possibly, there might be redundant pathways in place during maturation that are absent in germinated seedlings. Indeed ABI5 and other bZIP factors of the same class operate with many other TF in a complex combinatorial control of gene expression that is poorly understood (Nakamura et al., 2001; Lopez-Molina et al., 2002; Finkelstein et al., 2005; Cutler et al., 2010; Lindemose et al., 2013; Verdier et al., 2013). 
To characterize the part of the desiccome that is influenced by MtABI5 during the re-establishment of DT tolerance, a transcriptome analysis was performed on the protruded radicles of germinated Mtabi5 mutants before and after the

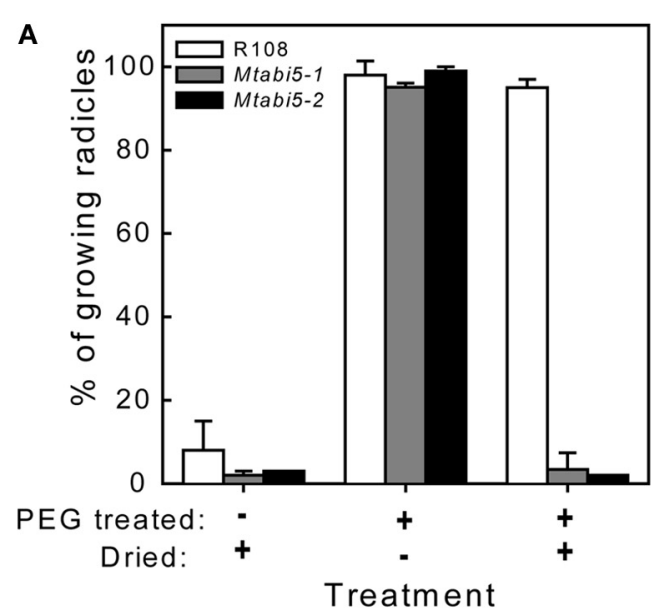

B

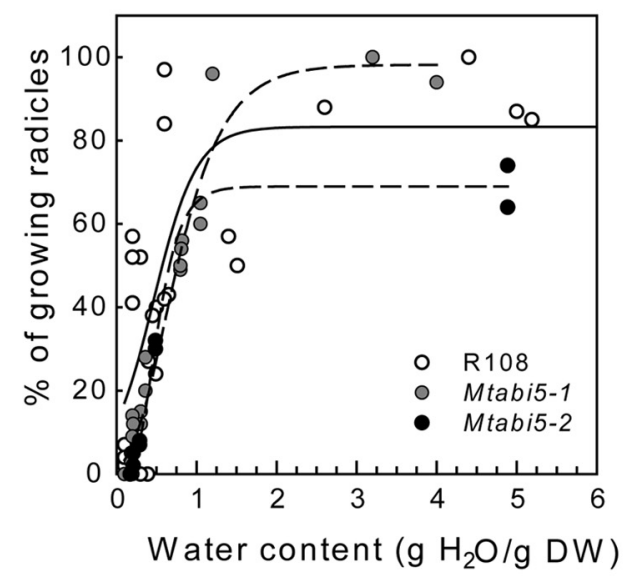

C

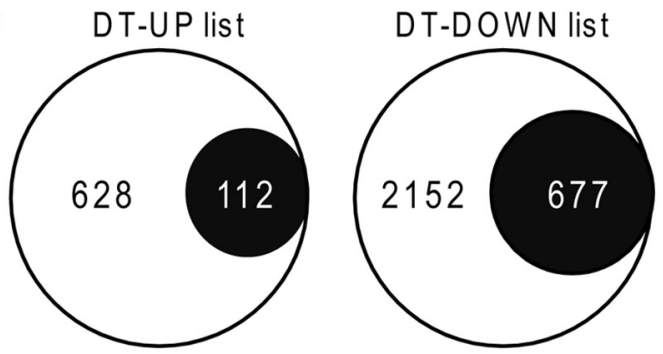

FIGURE 4 | Desiccation tolerance is not re-established during PEG treatment in germinated Mtabi5 seeds. (A) Percentages of desiccation tolerance of $2.7 \mathrm{~mm}$ long protruded radicles from germinated wild-type and Mtabi5 seeds. Seeds were dried at $42 \%$ relative humidity $(\mathrm{RH})$ for 3 days before or after a PEG treatment consisting in incubating seeds for 3 days in a polyethylene glycol (PEG) solution $(-1.7 \mathrm{MPa})$ at $10^{\circ} \mathrm{C}$. Data represent the average $( \pm \mathrm{SE})$ of three independent experiments of 50 seeds. (B) The effect of drying at $42 \% \mathrm{RH}$ at the indicated water content on survival of $2.7 \mathrm{~mm}$ long protruded radicles of germinated seeds. Each data points represent 30 seeds from three independent experiments. (C) Euler diagrams showing the number of transcripts in the DT-UP and DT-DOWN list (Figure 1) that do not change significantly in germinated Mtabi5 radicles after PEG incubation. incubation in the PEG solution (Figure 4C). A total of 852 out of the 3626 transcripts with increased levels in the wild type were no longer up-regulated by the PEG treatment in the Mtabi5 mutant $(P>0.01$ and ratio after PEG/before PEG $<1$ or $>-1)$. Likewise, out of the 6691 transcript levels that decreased during the PEG treatment in the wild type, 1865 no longer decreased in the Mtabi5 mutants. Next, these data were compared to the DT-UP and DT-DOWN lists (Figure 4C, Supplementary Table S2). 112 of the 740 transcripts (15\%) that are present in the DT-UP list were no longer up-regulated in the Mtabi5 mutants whereas 23\% (677) of the 2829 transcripts of DT-DOWN list were no longer down-regulated. From these data, $M t A B I 5$ seems to play a predominant role as a repressor rather than an activator during the re-establishment of DT. Further work is needed to investigate which of these genes are under the direct regulation of MtABI5 or modulated downstream of MtABI5 pathways.

\section{TRANSCRIPTOME ANALYSIS IDENTIFIES MtABI3-RELATED GENES INVOLVED IN DT}

A recent characterization of Tnt1 abi3 mutants of Medicago demonstrated that like for Arabidopsis, Medicago abi3 mutant seeds are desiccation sensitive and contain reduced amount of LEA proteins (Delahaie et al., 2013). To investigate which genes of the desiccome were regulated directly or acting downstream of $M t A B I 3$, we took advantage of recently published transcriptome data of developing desiccation-sensitive Mtabi3 mutant seeds (Verdier et al., 2013, GEO database accession GSE44291). These seeds were analyzed at 32 DAP and compared to wild type seeds of the same age, after DT had been acquired (Verdier et al., 2013). In addition, we compared this dataset to that of the transcriptome of Medicago roots ectopically expressing the genomic sequence of $M t A B I 3$ (35S::ABI3, Verdier et al., 2013; GEO database accession GSE44291). Amongst the 1151 transcripts with significantly decreased levels in the Mtabi3 seeds, 249 were present in DT-UP list (i.e., 33\% of the list, Figure 5, Supplementary Table S3) and 43 were present in the three data sets. Similarly, out 3745 transcripts that increased

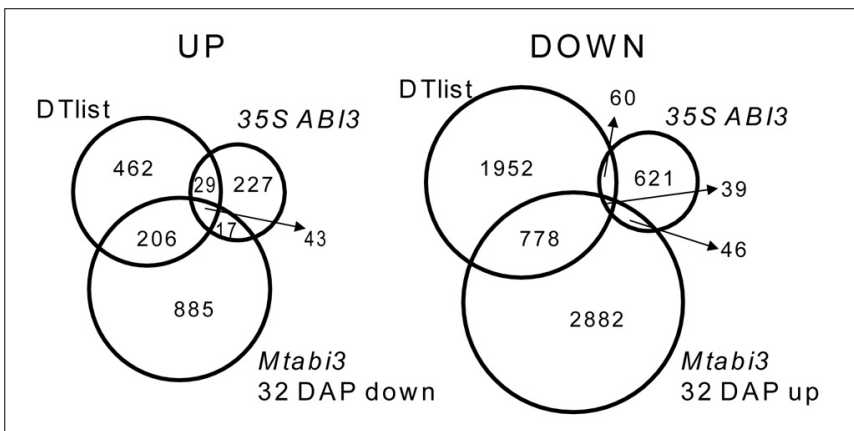

FIGURE 5 | Venn diagram identifying DT transcripts related to ABSCISIC ACID 3 (ABI3) of Medicago truncatula. Transcripts that were identified in the DT-UP or DT-DOWN lists were compared to those that are differentially expressed in Mtabi3 seeds at 32 DAP compared to wild type seeds (Mtabi3 32 DAP) and to those that are differentially expressed upon ectopic expression of $M t A B / 3$ in hairy roots compared to control. Transcript levels were considered significant when $P<0.01$ and $-1>\operatorname{Mlog} 2>1$. 
significantly in the Mtabi3 seeds, 817 were present in the DTDOWN list (i.e., 28\%) and 39 were found in common between the three data sets (Figure 5, Supplementary Table S3). To further validate the $M t A B I 3$-mediated expression of DT genes, we verified whether their Arabidopsis homologs were among the list of the experimentally validated targets of the Arabidopsis ABI3 regulon (Mönke et al., 2012). For the 43 up-regulated genes present in all three dataset (Figure 5), 19 Arabidopsis homologs were identified as part of the regulon of $M t A B I 3$. Genes in this list encoded LEA proteins and genes involved in seed storage deposition such as oleosins and seed storage proteins. It is noteworthy that transcripts of two cystathionine beta-synthase (CBS) domain proteins were also identified, one of them being $M t S N F 4 b$, a subunit of the metabolic regulator SUCROSE NONFERMENTING RELATD KINASE (SnRK1). $\mathrm{MtSNF} 4 \mathrm{~b}$ plays a role in seed longevity and activation of biotic stress pathways in dormant seeds (Rosnoblet et al., 2007; Bolingue et al., 2010). Since Mönke et al. (2012) did not investigate targets that are repressed by $\mathrm{ABI} 3$, a comparison was impossible (Figure 5).

\section{ANALYSIS OF ABI3- AND ABI5-SPECIFIC DATASETS IN RELATION TO DT}

Having identified the transcripts that are affected in the Mtabi3and Mtabi5 mutants in relation to DT, we investigated the putative function of the genes that modulated by one or both TFs (Figure 6). It should be noted that the change in transcript levels is not necessarily through a direct action of ABI3 and or ABI5, since transcript levels can be modified by downstream targets of these TFs or through other mechanisms than transcriptional activation. However, to facilitate the reading, we refer hereafter to $\mathrm{ABI} 3$ or $\mathrm{ABI} 5$-related genes. The putative regulation of transcripts by these two TFs is annotated in Table $\mathbf{1}$ and Supplemental Table 1. A GO-enrichment analysis was performed on these gene lists (Table 3).

Only a small amount of transcripts are influenced by both TFs, representing 4 and 7\% of the DT-UP and DT-DOWN lists, respectively (Figure 6). Among the 27 genes from the DT-UP list that are downstream of both TFs, we noticed three different glutamate-cysteine ligases that catalyze the first and rate limiting step in the biosynthesis of glutathione (GSH, Noctor et al., 2012) (Supplementary Table S1). In Arabidopsis, gsh1 mutants confer a recessive embryo-lethal phenotype and it was suggested that the autonomous synthesis of GSH in the embryo was necessary for proper seed maturation (Cairns et al., 2006). During drying, the resurrection plant Sporobolus stapfianus exhibited an important increase in GSH and $\gamma$-glutamyl amino acids compared to the desiccation-sensitive Sporobulus pyramidalis (Oliver et al., 2011). Thus, GSH might represent a key antioxidant involved for the survival in the dry state. A RT-qPCR study validated their expression level (Supplemental Table S4). Surprisingly, LEA transcripts were found to be regulated only by $\mathrm{ABI} 3$; in the Mtabi5 mutants they increased in a comparable way to the wild type radicles (Supplementary Table S3). Four TFs were related to both MtABI3 and MtABI5 (Table 1). It is noteworthy that homologs of two of them mediate light responses in Arabidopsis; FBH1 and a gene bearing high homology to LZF1 (LIGHT-REGULATED ZINC FINGER PROTEIN 1).

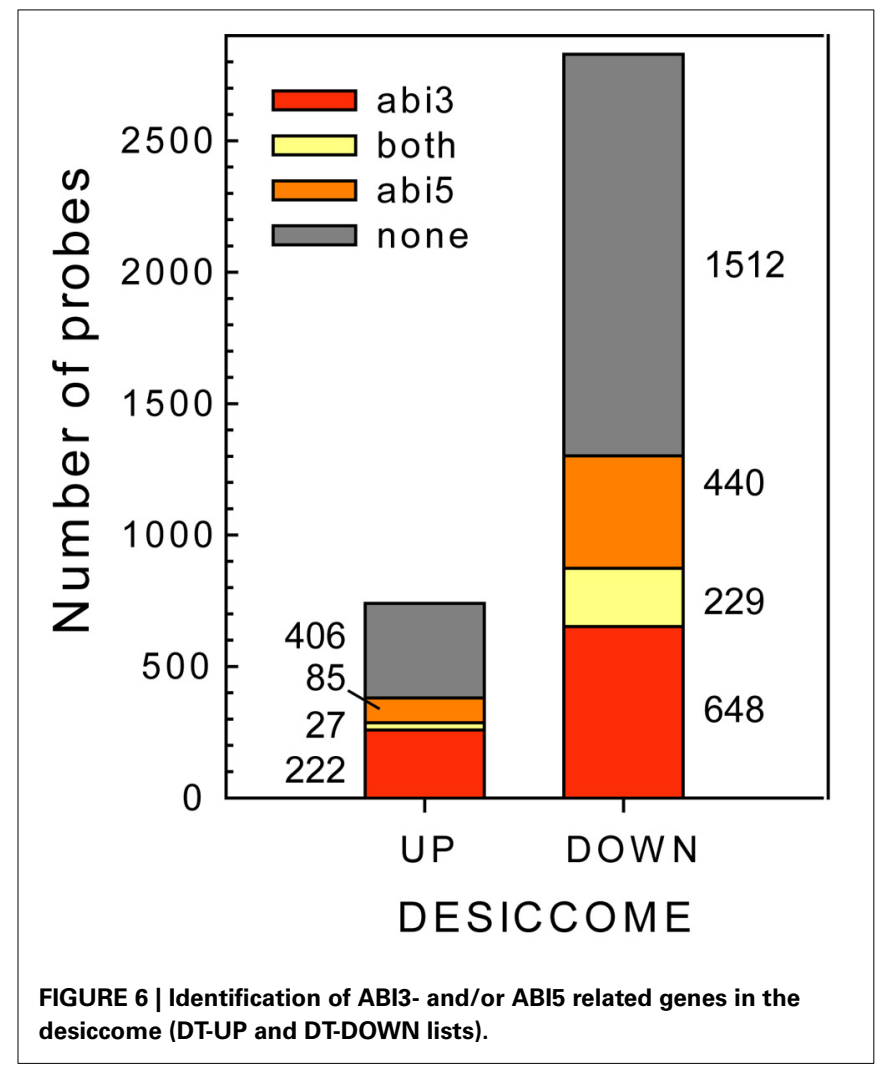

In Arabidopsis, LZF1 is a positive regulator functioning in deetiolation and the accumulation of anthocyanin (Chang et al., 2008).

The pathway downstream of $\mathrm{ABI} 3$ is more affected compared to ABI5, representing, respectively, 30\% (222 transcripts) of the DT-UP list and 23\% (648 transcripts) of DT-DOWN list. Biological processes that were overrepresented in the ABI3-related gene list were "response to abscisic acid stimulus," including ABA responsive genes such as homologs of RD26 or PROTEIN-LISOASPARTATE METHYLTRANSFERASE 1 (PIMT1), playing a role in protein repair after seed ageing (Ogé et al., 2008) (Table 3). The GO term "response to heat" included heat stress associated proteins, HSP (HSP101, sHPs) and HSF, whereas the GO term "multicellular organismal development" contained ABI3 targets, such as storage reserve proteins, 1-cys-peroxiredoxin and LEA genes.

Out of the 85 transcripts corresponding to Medicago genes that were identified as up-regulated via $M t A B I 5$, only few were annotated (Supplemental Tables S1, S2). Many of them corresponded to sequences implicated in transcriptional regulation (DNA polymerases, RNA processing) and post-transcriptional regulation (ubiquitin-related). Several bHLH TFs were identified, namely bHLH135 (PRE5), bHLH84, and bHLH130 (FBH4). The Arabidopsis FBH4 positively regulates CONSTANS transcription for photoperiodic flowering together with FBH1 (Ito et al., 2012).

A GO enrichment analysis on the DT-DOWN list revealed that the main biological processes for which transcripts are down-regulated by both TFs are cell cycle, secondary metabolic 
Table 3 | MtABI3 and MtABI5 are involved in complementary processes in relation to desiccation tolerance in Medicago truncatula.

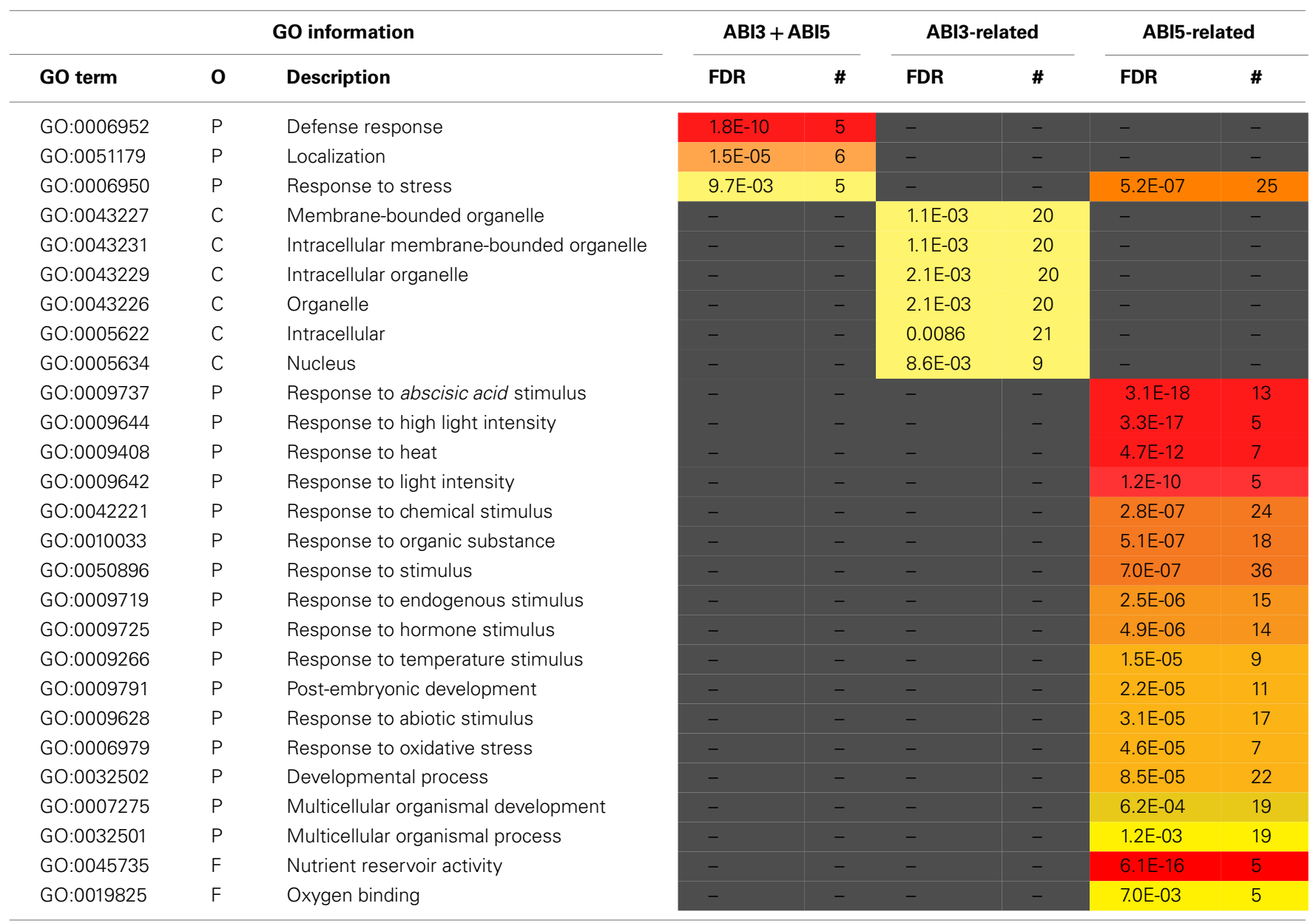

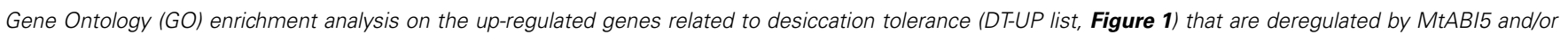

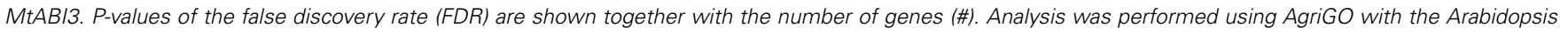
TAIR9 background applying a Chi ${ }^{2}$ statistical test with the Yekutieli multi-test adjustment method. P, Biological process; $C$, component; $F$, molecular function.

processes and specific kinase or receptor signaling pathways (Table 4). The ABI5-related dataset showed a highly significant overrepresentation of processes related to cell division and actin cytoskeleton organization. This category goes together with the previously mentioned CUC3 TF whose expression is affected by ABI5 and involved in the repression of cell division (Table 1). Another biological processes enriched in the ABI5-related probe list is "defense response," including genes involved in flavonoid and phenylpropanoid biosynthesis and programmed cell death. The GO analysis of the overrepresented biological processes of the down-regulated transcripts that are related to ABI3 is complementary to that of the ABI5related list (Table 4). ABI3 appears to regulate biological processes involved in "DNA replication," "cell wall modification" and genes involved in microtubule-based processes. Interestingly, this goes together with the ABI3-dependent up-regulation of PHS1 (PROPYZAMIDE-HYPERSENSITIVE 1), involved in phosphorylation cascades that control the dynamics of cortical microtubules in plant cells (Naoi and Hashimoto, 2004). Phs1 mutants are hypersensitive to ABA. In addition, other biological processes were related to primary metabolic process, such as fatty acid biosynthetic process, carbohydrate metabolic process, and lipid biosynthetic process. A final category is related to DNA repair.

\section{CONCLUDING REMARKS}

The transcriptome comparisons combining two developmental contexts during which DT is acquired, together with the transcriptome analysis of two desiccation sensitive mutants generated many intriguing hypotheses related to the regulatory mechanisms that determine DT. We are aware that the hypotheses described in this study are based solely on steady-state transcript levels and inferred from homologous genes characterized in Arabidopsis. Therefore, more work is necessary to define gene functions and dissect the complex regulation of gene expression. In addition, post-transcriptional and post-translational processes need to be taken into account. Yet, the data suggest that the regulatory pathways leading to DT act redundantly and respond to both developmental and environmental cues. None of the monogenic mutation in the 49 TF gene that were identified in the desiccome induced lethal or desiccation sensitive phenotypes in Arabidopsis, except for ABI3. These TFs are likely acting 
Table 4 | MtABI3 and MtABI5 are involved in complementary processes in relation to desiccation tolerance in Medicago truncatula.

\begin{tabular}{|c|c|c|c|c|c|c|c|}
\hline \multicolumn{2}{|r|}{ GO information } & \multicolumn{2}{|c|}{$A B I 3+A B I 5$} & \multicolumn{2}{|c|}{ ABI3-related } & \multicolumn{2}{|c|}{ ABI5-related } \\
\hline GO:0019748 & Secondary metabolic process & 3E-05 & 11 & 0.0092 & 12 & - & - \\
\hline GO:0051726 & Regulation of cell cycle & 0.0002 & 5 & - & - & 9E-05 & 9 \\
\hline GO:0007167 & Enzyme linked receptor protein signaling pathway & 0.0005 & 5 & - & - & $5 \mathrm{E}-07$ & 11 \\
\hline GO:0007166 & Cell surface receptor linked signaling pathway & 0.0049 & 5 & - & - & $4 \mathrm{E}-05$ & 11 \\
\hline GO:0009698 & Phenylpropanoid metabolic process & 0.0052 & 5 & $3 E-08$ & 10 & & \\
\hline GO:0051301 & Cell division & - & - & $2 \mathrm{E}-09$ & 8 & 0.0017 & 7 \\
\hline GO:0019438 & Aromatic compound biosynthetic process & - & - & $3 \mathrm{E}-08$ & 12 & - & - \\
\hline GO:0009699 & Phenylpropanoid biosynthetic process & - & - & $3 \mathrm{E}-08$ & 9 & - & - \\
\hline GO:0009832 & Plant-type cell wall biogenesis & - & - & $4 \mathrm{E}-06$ & 5 & - & - \\
\hline GO:0006950 & Response to stress & - & - & $4 \mathrm{E}-06$ & 44 & - & - \\
\hline GO:0006725 & Cellular aromatic compound metabolic process & - & - & $5 \mathrm{E}-06$ & 14 & - & - \\
\hline GO:0050896 & Response to stimulus & - & - & $5 \mathrm{E}-06$ & 65 & - & - \\
\hline GO:0042398 & Cellular amino acid derivative biosynthetic process & - & - & 1E-05 & 10 & 0.0045 & 11 \\
\hline GO:0006915 & Apoptosis & - & - & $2 \mathrm{E}-05$ & 8 & - & - \\
\hline GO:0009987 & Cellular process & - & - & $2 \mathrm{E}-05$ & 142 & 0.0097 & 202 \\
\hline GO:0012501 & Programmed cell death & - & - & $2 \mathrm{E}-05$ & 10 & - & - \\
\hline GO:0030029 & Actin filament-based process & - & - & $3 \mathrm{E}-05$ & 6 & - & - \\
\hline GO:0009813 & Flavonoid biosynthetic process & - & - & $4 \mathrm{E}-05$ & 5 & - & - \\
\hline GO:0042546 & Cell wall biogenesis & - & - & $5 \mathrm{E}-05$ & 5 & - & - \\
\hline GO:0048878 & Chemical homeostasis & - & - & 0.0023 & 6 & - & - \\
\hline GO:0050801 & Ion homeostasis & - & - & 0.0045 & 5 & - & - \\
\hline GO:0009617 & Response to bacterium & - & - & 0.0066 & 8 & - & - \\
\hline GO:0009605 & Response to external stimulus & - & - & 0.0092 & 11 & - & - \\
\hline GO:0032501 & Multicellular organismal process & - & - & 0.0092 & 33 & - & - \\
\hline GO:0000271 & Polysaccharide biosynthetic process & - & - & 0.0092 & 5 & - & - \\
\hline GO:0007275 & Multicellular organismal development & - & - & 0.0098 & 32 & - & - \\
\hline GO:0010876 & Lipid localization & - & - & - & - & $6 \mathrm{E}-24$ & 8 \\
\hline GO:0007017 & Microtubule-based process & - & - & - & - & $3 \mathrm{E}-11$ & 12 \\
\hline GO:0006259 & DNA metabolic process & - & - & - & - & 1E-09 & 23 \\
\hline GO:0007018 & Microtubule-based movement & - & - & - & - & $2 \mathrm{E}-09$ & 7 \\
\hline GO:0006260 & DNA replication & - & - & - & - & $5 \mathrm{E}-07$ & 10 \\
\hline GO:0042545 & Cell wall modification & - & - & - & - & $6 \mathrm{E}-05$ & 9 \\
\hline GO:0006261 & DNA-dependent DNA replication & - & - & - & - & $9 \mathrm{E}-05$ & 6 \\
\hline GO:0044238 & Primary metabolic process & - & - & - & - & 0.0004 & 170 \\
\hline GO:0005976 & Polysaccharide metabolic process & - & - & - & - & 0.0014 & 9 \\
\hline GO:0009664 & Plant-type cell wall organization & - & - & - & - & 0.0034 & 6 \\
\hline GO:0008152 & Metabolic process & - & - & - & - & 0.004 & 189 \\
\hline
\end{tabular}


Table 4 | Continued

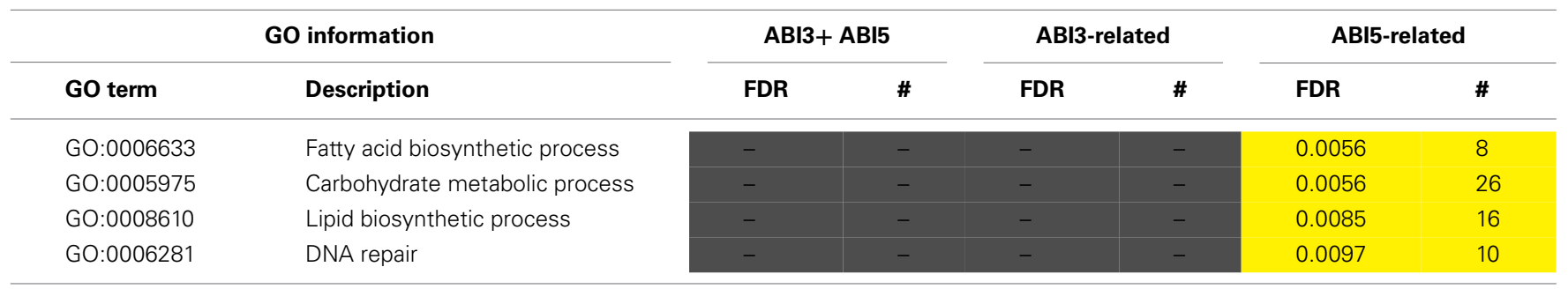

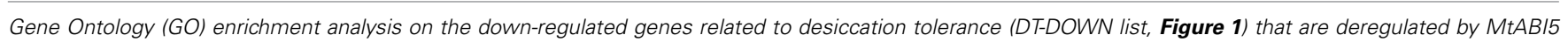

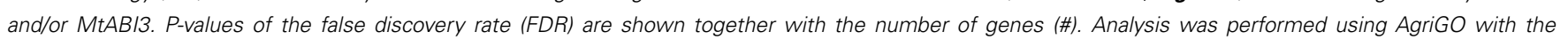

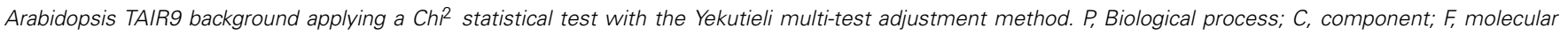
function.

redundantly depending of the environmental context. Our data suggest that $\mathrm{ABI} 3$ and ABI5 have complementary roles in DT and act in a complex combinatorial control of gene expression (Nakamura et al., 2001; Finkelstein et al., 2005; Cutler et al., 2010; Lindemose et al., 2013).

The desiccome contained a surprisingly high number of transcripts for which Arabidopsis homologs are involved in the control of flowering, cellular phase transitions and cell identity. These data suggest that DT evolved by coopting existing genetic pathways regulating developmental phase transition and light-sensing. There is increasing evidence in the literature suggesting an interaction between photoperiod and drought stress, whereby photoperiodically- or light-induced genes coordinate the ABA-mediated activation of genes (Chen et al., 2008; Riboni et al., 2013). Therefore, it is important to investigate whether genes regulated by photoperiod or light have a conserved function regulating DT both in seeds as well as resurrection plants.

\section{AUTHORS CONTRIBUTIONS}

Julia Buitink and Olivier Leprince conceived and designed the experiments and wrote the MS. Emmanuel Terrasson and David Lalanne performed the transcriptome analysis. Emmanuel Terrasson and Benoit Ly Vu carried out the physiological experiments. Julia Zinsmeister performed the qPCR experiments. Emmanuel Terrasson, Karima Righetti, and Sandra Pelletier performed the bioinformatic analyses. Emmanuel Terrasson and Karima Righetti critically reviewed the MS.

\section{ACKNOWLEDGMENTS}

Emmanuel Terrasson and this work were supported by a grant from the Région Pays-de-la-Loire (QUALISEM 2009-2013). We acknowledge Dr. Pascal Gamas and Jérôme Gouzy (INRA, Toulouse, France) for sharing the Medicago truncatula genome data. The abis mutants of Medicago truncatula used in this research project, which are jointly owned by the Centre National de la Recherche Scientifique, were obtained from The Samuel Roberts Noble Foundation, Inc. and were created through research funded, in part, by a grant from the National Science Foundation (NSF\# 703285).

\section{SUPPLEMENTARY MATERIALS}

The Supplementary Material for this article can be found online at: http://www.frontiersin.org/journal/10.3389/ fpls.2013.00497/abstract

Table S1 | List of transcripts that are differentially expressed in relation to desiccation tolerance (DT-UP and DT-DOWN) list (Figure 1).

Table S2 | List of desiccation tolerance (DT) correlated transcripts that are affected by the absence of MtABI5 compared to wild type seeds during the re-induction of DT.

Table S3 | List of transcripts that are affected by the absence of $M t A B I 3$ compared to wild type seeds during seed maturation at 32 DAP (Mtabi3 32 DAP), or that show increased or decreased transcript levels in hairy roots ectopically expressing $M t A B I 3$ compared to roots with an empty plasmid.

Table S4 | Transcript levels of two glutamyl cysteine ligase genes (Medtr5g010210.1 and Medtr5g010230.1) after the re-induction of desiccation tolerance in radicles of germinated wild type, Mtabi5-1 and Mtabi5-2 mutant seeds by a PEG treatment at $-1.7 \mathrm{MPa}$.

\section{REFERENCES}

Barrett, T., Troup, D. B., Wilhite, S. E., Ledoux, P., Evangelista, C., Kim, I. F., et al. (2012). NCBI GEO: archive for functional genomics data sets-10 years on. Nucleic Acids Res. 41, 991-995. doi: 10.1093/nar/gkq1184

Bartels, D., and Sunkar, R. (2005). Drought and salt tolerance in plants. Crit. Rev. Plant Sci. 24, 23-58. doi: 10.1080/07352680590 910410

Blödner, C., Goebel, C., Feussner, I., Gatz, C., and Polle, A. (2007). Warm and cold parental reproductive environments affect seed properties, fitness, and cold responsiveness in Arabidopsis thaliana progenies. Plant Cell Environ. 30, 165-175. doi: 10.1111/j.1365-3040.2006.01615.x

Bolingue, W., Rosnoblet, C., Ly Vu, B., Leprince, O., Aubry, C., and Buitink, J. (2010). MtSNF4b connects after-ripening and constitutive biotic defense responses in seeds of Medicago truncatula. Plant J. 61, 792-803. doi: 10.1111/j.1365-313X.2009.04106.x

Boudet, J., Buitink, J., Hoekstra, F. A., Rogniaux, H., Larré, C., Satour, P., et al. (2006). Comparative analysis of the heat stable proteome of radicles of Medicago truncatula seeds during germination identifies late embryogenesis abundant proteins associated with desiccation tolerance. Plant Physiol. 140, 1418-1436. doi: 10.1104/pp.105.074039

Buitink, J., Leger, J. L., Guisle, I., Ly Vu, B., Wuilleme, S., Lamirault, G., et al. (2006). Transcriptome profiling uncovers metabolic and regulatory processes occurring during the transition from desiccation sensitive to -tolerant stages in Medicago truncatula seeds. Plant J. 47, 735-750. doi: 10.1111/j.1365-313X.2006. 02822.x

Buitink, J., Ly Vu, B., Satour, P., and Leprince, O. (2003). A physiological model to study the re-establishment of desiccation tolerance in germinated radicles of Medicago truncatula Gaertn. seeds. Seed Sci. Res. 13, 273-286. doi: $10.1079 /$ ssr2003145 
Cairns, N. G., Pasternak, M., Wachter, A., Cobbett, C. S., and Meyer, A. J. (2006). Maturation of Arabidopsis seeds is dependent on gluthathione biosynthesis. Plant Physiol. 141, 446-455. doi: 10.1104/pp.106.077982

Chang, C.-S. J., Li, Y. H., Chen, L. T., Chen, W. C., Hsieh, W. P., Shin, J., et al. (2008). LZF1, a HY5-regulated transcriptional factor, functions in Arabidopsis de-etiolation. Plant J. 54, 205-219. doi: 10.1111/j.1365-313X.2008.0340

Chatelain, E., Hundertmark, M., Leprince, O., Le Gall, S., Satour, P., DelignyPenninck, S., et al. (2012). Temporal profiling of the heat-stable proteome during late maturation of Medicago truncatula seeds identifies a restricted subset of late embryogenesis abundant proteins associated with longevity. Plant Cell Environ. 35, 1440-1455. doi: 10.1111/j.1365-3040.2012.02501.x

Chen, H., Zhang, J., Neff, M. M., Hong, S. W., Zhang, H., Deng, X. W., et al. (2008). Integration of light and abscisic acid signalling during seed germination and early seedling development. Proc. Natl. Acad. Sci. U.S.A. 105, 4495-4500. doi: 10.1073/pnas.0710778105

Ciais, P., Reichstein, M., Viovy, N., Granier, A., Ogée, J., Allard, V., et al. (2005). Europe-wide reduction in primary productivity caused by the heat and drought in 2003. Nature 437, 529-533. doi: 10.1038/nature03972

Claeys, H., and Inzé, D. (2013). The agony of choice: how plants balance growth and survival under water-limiting conditions. Plant Physiol. 62, 1768-1779. doi: 10.1104/pp.113.220921

Cutler, S. R., Rodriguez, P. L., Finkelstein, R. R., and Abrams, S. R. (2010). Abscisic acid: emergence of a core signaling network. Annu. Rev. Plant Biol. 61, 651-679. doi: 10.1146/annurev-arplant-042809-112122

Delahaie, J., Hundertmark, M., Bove, J., Leprince, O., Rogniaux, H., and Buitink, J. (2013). LEA polypeptide profiling of recalcitrant and orthodox legume seeds reveals ABI3-regulated LEA protein abundance linked to desiccation tolerance. J. Exp. Bot. 64, 4559-4573. doi: 10.1093/jxb/ert274

Donohue, K. (2009). Completing the cycle: maternal effects as the missing link in plant life histories. Philos. Trans. R. Soc. B. 364, 1059-1074. doi: 10.1098/rstb.2008.0291

Du, Z., Zhou, X., Ling, Y., Zhang, Z. H., and Su, Z. (2010). AgriGO: a GO analysis toolkit for the agricultural community. Nucl. Acids Res. 38, W64-W70. doi: $10.1093 / \mathrm{nar} / \mathrm{gkq} 310$

Edgar, R., Domrachev, M., and Lash, A. E. (2002). Gene Expression Omnibus: NCBI gene expression and hybridization array data repository. Nucleic Acids Res. 30, 207-210. doi: 10.1093/nar/30.1.207

Faria, J. M. R., Buitink, J., van Lammeren, A. A. M., and Hilhorst, H. W. M. (2005). Changes in DNA and microtubules during loss and re-establishment of desiccation tolerance in germinating Medicago truncatula seeds. J. Exp. Bot. 56, 2119-2130. doi: 10.1093/jxb/eri210

Finkelstein, R. R., Gampala, S. S. L., Lynch, T. J., Thomas, T. L., and Rock, C. D. (2005). Redundant and distinct functions of the ABA response loci ABAINSENSITIVE(ABI)5 and ABRE-BINDING FACTOR (ABF)3. Plant Mol. Biol. 59, 253-267. doi: 10.1007/s11103-005-8767-2

Fujita, Y., Fujita, M., Shinozaki, K., and Yamaguchi-Shinozaki, K. (2011). ABAmediated transcriptional regulation in response to osmotic stress tolerance. J. Plant. Res. 124, 509-525. doi: 10.1007/s10265-011-0412-3

Gaff, D. F., and Oliver, M. (2013). The evolution of desiccation tolerance in angiosperm plants: a rare yet common phenomenon. Func. Plant Biol. 40, 315-328. doi: 10.1071/FP12321

Galinha, C., Hofhuis, H., Luijten, M., Willemsen, V., Blilou, I., Heidstra, R., et al. (2007). PLETHORA proteins as dose-dependent master regulators of Arabidopsis root development. Nature 449, 1053-1057. doi: 10.1038 /nature06206.

Gechev, T. S., Dinakar, C., Benina, M., Toneva, V., and Bartels, D. (2012). Molecular mechanisms of desiccation tolerance in resurrection plants. Cell. Mol. Life Sci. 69, 3175-3186. doi: 10.1007/s00018-012-1088-0

Gregis, V., Sessa, A., Colombo, L., and Kater, M. M. (2008). AGAMOUSLIKE24 and SHORT VEGETATIVE PHASE determine floral meristem identity in Arabidopsis. Plant J. 56, 891-902. doi: 10.1111/j.1365-313X.2008. 03648.x

Grierson, C., Du, J. S., Zabala, M. T., Beggs, K., Smith, C., Holdsworth, M., et al. (1994). Separate cis sequences and trans factors direct metabolic and developmental regulation of a potato tuber storage protein gene. Plant J. 5, 815-826. doi: 10.1046/j.1365-313X.1994.5060815.x

Hauser, F., Waadt, R., and Schroeder, J. I. (2011). Evolution of abscisic acid synthesis and signalling mechanisms. Curr. Biol. 21, 346-355. doi: 10.1016/j.cub.2011.03.015
Hibara, K., Karim, M. R., Takada, S., Taoka, K., Furutani, M., Aida, M., et al. (2006). Arabidopsis CUP-SHAPED COTYLEDON3 regulates postembryonic shoot meristem and organ boundary formation. Plant Cell. 18, 2946-2957. doi: 10.1105/tpc.106.045716

Hirayama, T., and Shinozaki, K. (2010). Research on plant abiotic stress responses in the post-genome era: past, present and future. Plant J. 61, 1041-1052. doi: 10.1111/j.1365-313X.2010.04124.x

Hoekstra, F. A., Golovina, E. A., and Buitink, J. (2001). Mechanisms of plant desiccation tolerance. Trends Plant Sci. 6, 431-438. doi: 10.1016/S13601385(01)02052-0

Ito, S., Song, Y. H., Josephson-Day, A. R., Miller, R. J., Breton, G., Olmstead, R. G., et al. (2012). FLOWERING BHLH transcriptional activators control expression of the photoperiodic flowering regulator CONSTANS in Arabidopsis. Proc. Natl. Acad. Sci. U.S.A. 109, 3582-3587. doi: 10.1073/pnas.1118876109

Jang, S., Torti, S., and Coupland, G. (2009). Genetic and spatial interactions between FT, TSF and SVP during the early stages of floral induction in Arabidopsis. Plant J. 60, 614-625. doi: 10.1111/j.1365-313X.2009.03986.x

Kim, D. H., Yamaguchi, S., Lim, S., Oh, E., Park, J., Hanada, A., et al. (2008). SOMNUS, a CCCH-type Zinc finger protein in Arabidopsis, negatively regulates light-dependent seed germination downstream of PIL5. Plant Cell 20, 1260-1277. doi: 10.1105/tpc.108.058859.

Kochanek, J., Steadman, K. J., Probert, R. J., and Adkins, S. W. (2011). Parental effects modulate seed longevity: exploring parental and offspring phenotypes to elucidate pre-zygotic environmental influences. New Phytol. 191, 223-233. doi: 10.1111/j.1469-8137.2011.03681

Leprince, O., and Buitink, J. (2010). Desiccation tolerance: from genomics to the field. Plant Sci. 179, 554-564. doi: 10.1016/j.plantsci.2010.02.011

Lindemose, S., O'Shea, C., Jensen, M. K., and Skriver, K. (2013). Structure, function and networks of transcription factors involved in abiotic stress responses. Int. J. Mol. Sci. 14, 5842-5878. doi: 10.3390/ijms14035842

Lopez-Molina, L., Mongrand, S., and Chua, N. H. (2001). A postgermination developmental arrest checkpoint is mediated by abscisic acid and requires the ABI5 transcription factor in Arabidopsis. Proc. Natl. Acad. Sci. U.S.A. 98, 4782-4787. doi: 10.1073/pnas.081594298

Lopez-Molina, L., Mongrand, S., McLachlin, D. T., Chait, B. T., and Chua, N. H. (2002). ABI5 acts downstream of ABI3 to execute an ABA-dependent growth arrest during germination. Plant J. 32, 317-328. Doi: 10.1046/j.1365313X.2002.01430.x

Maia, J., Dekkers, B. J. W., Provart, N. J., Ligterink, W., and Hilhorst, H. W. M. (2011). The re-establishment of desiccation tolerance in germinated Arabidopsis thaliana seeds and its associated transcriptome. Plos ONE 6:e29123. doi: 10.1371/journal.pone.0029123

Martinez, I. M., and Chrispeels, M. J. (2003). Genomic analysis of the unfolded protein response in Arabidopsis shows its connection to important cellular processes. Plant Cell 15, 561-576. doi: 10.1105/tpc.007609

Mönke, G., Seifert, M., Keilwagen, J., Mohr, M., Grosse, I., Hähnel, U., et al. (2012). Toward the identification and regulation of the Arabidopsis thaliana ABI3 regulon. Nucleic Acids Res. 40, 8240-8254. doi: 10.1093/nar/gks594

Moore, J. P., Tuan, Le, N., Brandt, W. F., Driouich, A., and Farrant, J. M. (2009). Towards a systems-based understanding of plant desiccation tolerance. Trends Plant Sci. 14, 110-117. doi: 10.1016/j.tplants.2008.11.007

Moore, J. P., Vicre-Gibouin, M., Farrant, J. M., and Driouich, A. (2008). Adaptations of higher plant cell walls to water loss: drought vs. desiccation. Physiol. Plant 134, 237-245. doi: 10.1111/j.1399-3054.2008.01134.x

Nakabayashi, K., Okamoto, M., Koshiba, T., Kamiya, Y., and Nambara, E. (2005). Genome-wide profiling of stored mRNA in Arabidopsis thaliana seed germination: epigenetic and genetic regulation of transcription in seed. Plant J. 41, 697-709. doi: 10.1111/j.1365-313X. 2005.02337.x

Nakamura, S., Lynch, T. J., and Finkelstein, R. R. (2001). Physical interactions between ABA response loci of Arabidopsis. Plant J. 26, 627-635. doi: 10.1046/j.1365-313x.2001.01069.x

Nakashima, K., Fujita, Y., Katsura, K., Maruyama, K., Narusaka, Y., Seki, M., et al. (2006). Transcriptional regulation of ABI3- and ABA responsive genes includeing RD29B and RD29A in seeds, germinating embryos, and seedlings of Arabidopsis. Plant Mol. Biol. 60, 51-68. doi: 10.1007/s11103-005-2418-5

Nakashima, K., and Yamaguchi-Shinozaki, K. (2013). ABA signaling in stress-response and seed development. Plant Cell Rep. 32, 959-970. doi: 10.1007/s00299-013-1418-1 
Naoi, K., and Hashimoto, T. (2004). A semidominant mutation in an Arabidopsis mitogen-activated protein kinase phosphatase-like gene compromises cortical microtubule organization. Plant Cell 16, 1841-1853. doi: 10.1105/tpc. 021865

Noctor, G., Mhamdi, A., Chaouch, S., Han, Y., Neukermans, J., Marquez-Garcia, B., et al. (2012). Glutathione in plants: an integrated overview. Plant Cell Environ. 35, 454-484. doi: 10.1111/j.1365-3040.2011.02400.x

Ogé, L., Bourdais, G., Bove, J., Collet, B., Godin, B., Granier, F., et al. (2008). Protein repair L-isoaspartyl methyltransferase 1 is involved in both seed longevity and germination vigor in Arabidopsis. Plant Cell 20, 3022-3037. doi: 10.1105/tpc.108.058479

Oliver, M. J., Guo, L., Alexander, D. C., Ryals, J. A., Wone, B. W. M., and Cushman, J. C. (2011). A sister group constrast using untargeted global metabolomic analysis delineates the biochemical regulation underlying desiccation tolerance in Sporobulus stapfianus. Plant Cell 23, 1231-1248. doi: 10.1105/tpc.110. 082800 .

Ooms, J. J. J., Kloorsterziel, L. K. M., Bartels, D., Koorneef, M., and Karssen, C. M. (1993). Acquisition of desiccation tolerance and longevity in seeds of Arabidopsis thaliana - A comparative study using abscisic acid-insensitive abi3 mutants. Plant Physiol. 102, 1185-1191. doi: 10.1104/pp.102.4.1185

Page, R. D. M. (1996). Tree View: an application to display phylogenetic trees on personal computers. Comput. Appl. Biosci. 12, 357-358. doi: 10.1093/bioinformatics/12.4.357

Pagnussat, G. C., Yu, H. J., Ngo, Q. A., Rajani, S., Mayalagu, S., Johnson, C. S., et al. (2005). Genetic and molecular identification of genes required for female gametophyte development and function in Arabidopsis. Development 32, 603-614. doi: 10.1242/dev.01595

Riboni, M., Galbiati, M., Tonelli, C., and Conti, L. (2013). GIGANTEA enables drought escape response via abscicic acid-dependent activation of florigens and SUPPRESSOR OF OVEREXPRESSION OF CONSTANS. Plant Physiol. 162, 1706-1719. doi: 10.1104/pp.113.217729

Rodriguez, M. C. S., Edsgärd, D., Hussain, S. S., Alquezar, D., Rasmussen, M., Gilbert, T., et al. (2010). Transcriptomes of the desiccation-tolerant resurrection plant Craterostigma plantagineum. Plant J. 63, 212-228. doi: 10.1111/j.1365313X.2010.04243.x.

Rosnoblet, C., Aubry, C., Leprince, O., Ly Vu, B., Rogniaux, H., and Buitink, J. (2007). The regulatory gamma subunit SNF4b of the sucrose nonfermenting related kinase complex is involved in longevity and stachyose accumulation during maturation of Medicago truncatula seeds. Plant J. 51, 47-59. doi: 10.1111/j.1365-313X.2007.03116.x

Tunnacliffe, A., and Wise, M. J. (2007). The continuing conundrum of LEA proteins. Naturwissenschaften 94, 791-812. doi: 10.1007/s00114-007-0254-y

Turchi, L., Carabelli, M., Ruzza, V., Possenti, M., Sassi, M., Penalosa, A., et al. (2013). Arabidopsis HD-Zip II transcription factors control apical embryo development and meristem function. Development 140, 2118-2129. doi: 10.1242/dev.092833

Verdier, J., Lalanne, D., Pelletier, S., Torres-Jerez, I., Righetti, K., Bandyopadhyay, K., et al. (2013). A regulatory network-based approach dissects late maturation processes related to the acquisition of desiccation tolerance and longevity of Medicago truncatula seeds. Plant Physiol. 163, 757-774. doi: 10.1104/pp.113.222380

Yano, R., Kanno, Y., Jikumaru, Y., Nakabayashi, K., Kamiya, Y., and Nambara, E. (2009). CHOTTO1, a putative double APETALA2 repeat transcription factor, is involved in abscisic acid-mediated repression of gibberellin biosynthesis during seed germination in Arabidopsis. Plant Physiol. 151, 641-654. doi: 10.1104/pp.109.142018

Yotsui, I., Saruhashi, M., Kawato, T., Taji, T., Hayashi, T., Quatrano, R. S., et al. (2013). ABSCISIC ACID INSENSITIVE3 regulates abscissic acidresponsive gene expression with the nuclear factor $\mathrm{Y}$ complex through the ACTT-core element in Physcomitrella patens. New Phytol. 199, 101-109. doi: 10.1111/nph.12251

Zhang, Y., Cao, G., Qu, L. J., and Gu, H. (2009). Characterization of Arabidopsis MYB transcription factor gene AtMYB17 and its possible regulation by LEAFY and AGL15. J. Genet. Genomics. 36, 99-107. doi: 10.1016/S16738527(08)60096-X

Zhao, Y., Hu, Y., Dai, M., Huang, L., and Zhou, D. X. (2009). The WUSCHELrelated homeobox gene WOX11 is required to activate shoot-borne crown root development in rice. Plant Cell 21, 736-748. doi: 10.1105/tpc.108.061655

Conflict of Interest Statement: The authors declare that the research was conducted in the absence of any commercial or financial relationships that could be construed as a potential conflict of interest.

Received: 29 September 2013; accepted: 18 November 2013; published online: 11 December 2013.

Citation: Terrasson E, Buitink J, Righetti K, Ly Vu B, Pelletier S, Zinsmeister J, Lalanne $D$ and Leprince $O$ (2013) An emerging picture of the seed desiccome: confirmed regulators and newcomers identified using transcriptome comparison. Front. Plant Sci. 4:497. doi: 10.3389/fpls.2013.00497

This article was submitted to Plant Physiology, a section of the journal Frontiers in Plant Science.

Copyright (C) 2013 Terrasson, Buitink, Righetti, Ly Vu, Pelletier, Zinsmeister, Lalanne and Leprince. This is an open-access article distributed under the terms of the Creative Commons Attribution License (CC BY). The use, distribution or reproduction in other forums is permitted, provided the original author(s) or licensor are credited and that the original publication in this journal is cited, in accordance with accepted academic practice. No use, distribution or reproduction is permitted which does not comply with these terms. 